\title{
CHARACTERIZATION OF THE EFFECTS OF A SUN-SYNCHRONOUS ORBIT SLOT ARCHITECTURE ON THE EARTH'S ORBITAL DEBRIS ENVIRONMENT
}

\author{
A Thesis \\ Presented to \\ the Faculty of California Polytechnic State University \\ San Luis Obispo
}

\author{
In Partial Fulfillment \\ of the Requirements for the Degree \\ Master of Science in Aerospace Engineering
}

by

Connor D. Noyes

June 2013 
(C) 2013

Connor D. Noyes

ALL RIGHTS RESERVED 


\section{COMMITTEE MEMBERSHIP}

TITLE:

Characterization of the Effects of a SunSynchronous Orbit Slot Architecture on the Earth's Orbital Debris Environment

AUTHOR: Connor D. Noyes

DATE SUBMITTED: June 2013

COMMITTEE CHAIR: Kira J. Abercromby, Ph.D.

Professor of Aerospace Engineering

COMMITTEE MEMBER: Karl D. Bilimoria, Ph.D.

NASA Ames Research Scientist

COMMITTEE MEMBER: David B. Esposto

Aerospace Engineering Lecturer

COMMitTEE MEMBER: Gary B. Hughes, Ph.D.

Professor of Statistics 


\begin{abstract}
Characterization of the Effects of a Sun-Synchronous Orbit Slot Architecture on the Earth's Orbital Debris Environment

Connor D. Noyes

Low Earth orbit represents a valuable limited natural resource. Of particular interest are sun-synchronous orbits; it is estimated that approximately $44 \%$ of low Earth satellites are sun-synchronous. A previously developed sun-synchronous orbit slot architecture is considered. An in-depth analysis of the relative motion between satellites and their corresponding slots is performed. The long-term evolution of Earth's orbital environment is modeled by a set of coupled ordinary differential equations. A metric for quantifying the benefit, if any, of implementing a sun-synchronous architecture is developed. The results indicate that the proposed slot architecture would reduce the frequency of conjunctions and collisions between satellites in sun-synchronous orbits.
\end{abstract}




\section{Acknowledgments}

I would first and foremost like to thank my mother without whom none of this would have been possible.

To my graduate advisor Dr. Kira Abercromby, words cannot express how grateful I am. My deepest thanks for igniting my interest in astrodynamics, for writing me countless letters of recommendation, for encouraging me to seek higher education, and for so much more.

I am indebted to Dr. Karl Bilimoria for consistently guiding my research. I can only hope to one day be as talented a research scientist as he has demonstrated himself to be. I would also like to express my gratitude to Dr. Gary Hughes for his relentless enthusiasm, and for bringing me up to speed in the field of statistics which, but for his help, I was woefully deficient. Thank you to Dave Esposto for his practical insights into the complexities of spacecraft mission design.

Thank you to everyone at Ames Research Center, the University Affiliated Research Center, the Systems Teaching Institute, and the San Jose State University Research Foundation, including, but certainly not limited to, Dr. Bassam Mussafar, Dr. Olenka Hubickyj, and Ms. Jennifer Victoria for showing me that NASA truly is a remarkable organization filled with remarkable people. This research was made possible by funding from the UARC System Teaching Institute.

I would also like to thank Analytical Graphics, Inc. for allowing me the use of their Systems Tool Kit software.

Finally, I dedicate this thesis to the loving memory of my father who provided me all the skills necessary to succeed in life. 


\section{Contents}

List of Tables viii

List of Figures $\quad$ ix

1 Introduction 1

1.1 Sun-Synchronous Orbit . . . . . . . . . . . . . . . . . . . 1

2 Slot Architecture Development 4

3 Relative Motion Analysis $\quad 7$

3.1 Method of Analysis . . . . . . . . . . . . . . 8

3.2 Conformance Criterion .................. . . 9

3.3 Relative Motion Results . . . . . . . . . . . . . . . . . . . . . 11

3.3.1 Gravity Only .................. 11

3.3.2 Gravity and Atmospheric Drag . . . . . . . . . . . 14

4 Orbital Debris Environment Model 20

4.1 Model Additions and Modifications . . . . . . . . . . . . . . . 22

4.1.1 Extension to Additional Altitudes . . . . . . . . . . . 22

4.1.2 Decay into the Shell of Interest . . . . . . . . . . . . 23

4.1 .3 Parameter Growth . . . . . . . . . . . . . . . 24

4.2 The Environment Model . . . . . . . . . . . . . . 25

4.2.1 Model Validation ................. . 26

4.2.2 SOI Discretization Sensitivity Analysis . . . . . . . . . 28

5 Numerical Results $\quad 30$

5.1 Baseline Scenario . . . . . . . . . . . . . . . . . . . 30 
5.1.1 Parameter Estimation . . . . . . . . . . . . 30

5.2 No Active Spacecraft Collisions Scenario . . . . . . . . . . . . . . 34

5.3 No SSO Collisions Scenario _. . . . . . . . . . . . . 34

5.4 Results and Discussion . . . . . . . . . . . . . 35

6 Concluding Remarks $\quad 42$

7 Future Work 44

$\begin{array}{ll}\text { Bibliography } & 46\end{array}$ 


\section{List of Tables}

5.1 Key model parameters as they vary with shell of interest . . . . . 33

5.2 Percent reduction in number of active spacecraft destroyed relative to baseline case due to full compliance with the slot architecture for various model configurations . . . . . . . . . . . . . . . 41 


\section{List of Figures}

1.1 Mean Altitude vs inclination of the LEO environment with SSO highlighted . . . . . . . . . . . . . . . 3

2.1 Architecture employed at a single altitude $\ldots \ldots \ldots$. . . . 6

3.1 Representation of the conformance criterion for scenarios involving atmospheric drag . . . . . . . . . . . . . . . 11

3.2 Motion of a satellite at $800 \mathrm{~km}$ altitude relative to its slot over one day propagated with the effects of $J_{2} \ldots \ldots \ldots \ldots$

3.3 Motion of a satellite at $800 \mathrm{~km}$ altitude relative to its slot over six months propagated with the effects of $J_{2} \ldots \ldots \ldots$

3.4 Motion of a satellite at $800 \mathrm{~km}$ altitude relative to its slot over one day propagated with the $70 x 70$ gravity field . . . . . . . . . 14

3.5 Motion of a satellite at $800 \mathrm{~km}$ altitude relative to its slot over six months propagated with the $70 \times 70$ gravity field . . . . . . . 15

3.6 Conformance times for gravity-only scenarios . . . . . . . . . 16

3.7 Motion of a satellite at $800 \mathrm{~km}$ altitude relative to its slot over ten days propagated with the 70x70 gravity field and atmospheric drag 17

3.8 Along-track separation of a satellite at $800 \mathrm{~km}$ during solar maximum conditions . . . . . . . . . . . . . . . . 18

3.9 Along-track separation of a satellite at $800 \mathrm{~km}$ during solar minimum conditions . . . . . . . . . . . . . . . . 18

3.10 Worst-case estimate of conformance time vs altitude for satellites propagated with 70x70 gravity field model and atmospheric drag .

4.1 Fragment growth over time . . . . . . . . . . . . . 27

4.2 Total fragments and intacts over time . . . . . . . . . . 27 
4.3 Comparison of the evolution of hazardous and benign fragments for two different spatial discretizations . . . . . . . . . . 28

5.1 Density per latitude bin of the current space environment . . . . 35

5.2 Density per latitude bin of the current space environment without active SSO satellites . . . . . . . . . . . . . . . 36

5.3 Number of intact-on-intact collisions and total number of active spacecraft destroyed . . . . . . . . . . . . . . 37

5.4 Evolution of hazardous and benign fragments . . . . . . . 38

5.5 Evolution of hazardous and benign fragments . . . . . . . 38

5.6 Evolution of hazardous and benign fragments . . . . . . . . 39

5.7 Cumulative number of intact-on-intact collisions and spacecraft destroyed with no collision avoidance . . . . . . . . . . . 40 


\section{List of Acronyms}

$\begin{array}{ll}\text { ECI } & \text { Earth-Centered Inertial } \\ \text { GEO } & \text { Geosyncronous Earth Orbit } \\ \text { HPOP } & \text { High Precision Orbit Propagator } \\ \text { JSpOC } & \text { Joint Space Operations Center } \\ \text { LEO } & \text { Low Earth Orbit } \\ \text { MLT } & \text { Mean Local Time } \\ \text { NASA } & \text { National Aeronautics and Space Administration } \\ \text { ODE } & \text { Ordinary Differential Equation } \\ \text { RAAN } & \text { Right Ascension of the Ascending Node } \\ \text { SFU } & \text { Solar Flux Unit } \\ \text { SOI } & \text { Shell Of Interest } \\ \text { STK } & \text { Systems Tool Kit } \\ \text { STM } & \text { Space Traffic Management } \\ \text { SSO } & \text { Sun-Synchronous Orbit } \\ \text { TLE } & \text { Two Line Element set } \\ \text { UCS } & \text { Union of Concerned Scientists }\end{array}$




\section{List of Symbols}

$\begin{array}{ll}a & \text { semi-major axis } \\ \frac{A}{m} & \text { area to mass ratio } \\ e & \text { eccentricity } \\ F(t) & \text { number of fragments } \\ i & \text { inclination } \\ J_{2} & \text { second-order zonal harmonic } \\ R(t) & \text { number of rocket bodies } \\ R_{\oplus} & \text { radius of the Earth } \\ S_{d}(t) & \text { number of active spacecraft with deorbit capability } \\ S_{n}(t) & \text { number of active spacecraft without deorbit capability } \\ S_{n}^{o}(t) & \text { number of inactive spacecraft } \\ u & \text { argument of latitude } \\ \beta & \text { collision rate parameter } \\ \delta & \text { fragment generation parameter } \\ \eta & \text { launch rate escalation factor } \\ \theta_{d} & \text { fraction of future spacecraft with deorbit capability } \\ \lambda(t) & \text { rate at which spacecraft are launched into an SOI } \\ \lambda_{F} & \text { rate at which fragments decay into an SOI } \\ \lambda_{R} & \text { rate at which non-deorbiting rocket bodies launched into an SOI } \\ \mu & \text { gravitational parameter of the Earth } \\ \mu_{F} & \text { rate at which fragments decay out of an SOI } \\ \mu_{n} & \text { natural spacecraft decay rate } \\ \mu_{o} & \text { inverse of average operational spacecraft lifetime } \\ \mu_{R} & \text { natural rocket body decay rate } \\ \nu & \text { true anomaly } \\ \Omega & \text { right ascension of the ascending node } \\ \omega & \text { argument of perigee } \\ & \end{array}$




\section{Chapter 1}

\section{Introduction}

The number of debris objects in orbit around Earth has risen steadily since first Man became capable of launching satellites into space.[1] Today, there are more than a thousand active satellites, over 22,000 debris objects with characteristic length greater than $10 \mathrm{~cm}$, and millions of smaller pieces of debris in Earth orbit.[2] Nearly 50\% of the active satellites and a majority of debris objects are in low Earth orbit (LEO), i.e. in an orbit below $2000 \mathrm{~km}$ altitude.[3, 4] This congestion frequently results in close approaches and occasionally actual collisions between LEO objects as was the case in February 2009 when nonoperational satellite Cosmos collided with active satellite Iridium [1], producing approximately a thousand pieces of debris larger than $10 \mathrm{~cm}$.

\subsection{Sun-Synchronous Orbit}

Based on analysis of the unclassified catalog of satellites available through Space Track, it is estimated that $44 \%$ of active LEO satellites are in sun-synchronous orbit (SSO).[2] Sun-synchronous orbit is achieved by exploiting the perturbing 
effect of $J_{2}$ on $\Omega$, right ascension of the ascending node (RAAN).[5] A satellite is in SSO if its nodal rate matches the average rate of the Sun's motion around the Earth, i.e., when

$$
\dot{\Omega}=\dot{\Omega}_{\text {SunSyn }}=\frac{1 \text { rev }}{1 \text { year }}=\frac{360^{\circ}}{365.242 \text { days }}
$$

where $\dot{\Omega}$ is the time rate of change of $\Omega$, and $\dot{\Omega}_{\text {SunSyn }}$ is the ideal value of $\dot{\Omega}$ for achieving sun-synchronicity. This is accomplished through a combination of semi-major axis (a), eccentricity (e), and orbital inclination (i). Typically the altitude of a satellite is dependent upon its mission and the eccentricity of a LEO satellite is often chosen to be near-zero.[5] The required inclination can then be solved for as

$$
i=\cos ^{-1}\left(\frac{-2 a^{7 / 2} \dot{\Omega}_{\text {SunSyn }}\left(1-e^{2}\right)^{2}}{3 R_{\oplus}^{2} J_{2} \sqrt{\mu}}\right)
$$

where $R_{\oplus}$ is the radius of the Earth, $J_{2}$ is the second-order zonal harmonic, and $\mu$ is the gravitational parameter of the Earth.[5] The angle between the orbital plane and the line between Earth and the Sun is determined by the satellite's $\Omega$ value.

The constant illumination resulting from SSO is desirable for a number of reasons ranging from wanting a constant source of radiation for solar panels to maintaining a constant viewing condition for Earth-observing satellites. [5, 6] Consider, then, that with $44 \%$ of LEO satellites in $\mathrm{SSO}$, [2] the region is a popular choice for mission designers and may soon become overcrowded. Compound this with the fact that collision probabilities are greatest when the sum of two satellites' inclinations is near $180^{\circ},[1]$ as is the case for SSOs, and the risk of collision becomes quite high among this class of satellites. Figure 1.1 shows mean altitude versus inclination for the current distribution of LEO satellites 
with those in SSO highlighted.

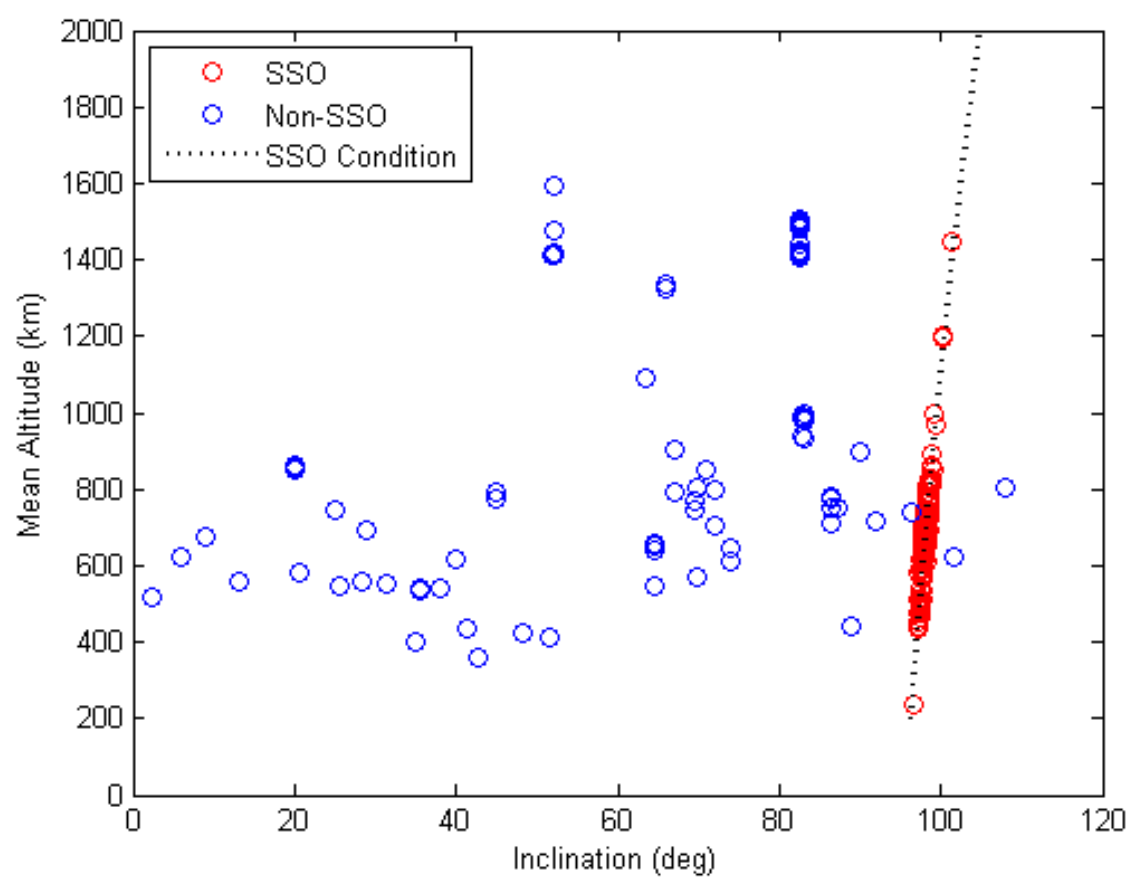

Figure 1.1: Mean Altitude vs inclination of the LEO environment with SSO highlighted

Currently, the are no operational guidelines or restrictions for launches into SSO. Close approaches, often referred to as conjunctions, are not uncommon among LEO satellites and commonly result in avoidance maneuvers. Past research has been done into an approach to keep this regime better separated both horizontally $[2,7]$ and vertically.[8] Chapter 2 reviews a portion of this effort. Previous works neither claimed an immediate need nor placed judgment upon the architecture; the later chapters of this work analyze the benefits that can be gleamed from its implementation by analyzing the evolution of orbital debris environment at a macroscopic level. 


\section{Chapter 2}

\section{Slot Architecture Development}

Due to the problems discussed in chapter 1, space traffic management (STM) is becoming a burgeoning field. With regard to SSO specifically, the last few years have seen a number of articles, conference proceedings, and Master's theses with ideas and potential solutions to the frequent conjunctions and the avoidance maneuvers they often necessitate. $[2,6-8]$ One such idea that has gained traction is a slot architecture designed to separate SSOs while avoiding placing unnecessarily stringent or taxing requirements on the satellites. This chapter aims to give a brief overview of the recent iterations of the architecture, particularly those of Ref.[2, 7], for the purpose of self-sufficiency.

The slot architecture concept is similar in nature to the way in which geosyn-

chronous (GEO) satellites are separated by longitude. GEO satellites are required to maintain orbit within their designated longitude bin and perform a stationkeeping maneuver should they drift outside. Unlike GEO, there are no restrictions on launching into LEO, nor are there similar standards for station-keeping and trajectory maintenance. A more complex method of separation is required for SSOs because SSO satellites occur over a range of altitudes unlike GEO satellites 
which require a very specific altitude and generally have near-zero inclinations and eccentricities. The dynamics of the situation are therefore more complicated when dealing with SSOs.

The architecture defines slots which are imaginary points in space that move according to idealized motion,i.e., slots are not subjected to all of the perturbing forces that satellites actually experience. At each altitude, a constellation is formed by the slots which are guaranteed to be well separated due to their simplified dynamics. An SSO satellite is then assigned to a slot that suits its mission requirements. The satellites will move relative to the slots due to the difference in their dynamics; satellites would theoretically be required to perform maintenance maneuvers whenever they no longer are in compliance with the architecture. The criteria for compliance is defined later based on the relative motion analysis of chapter 3 , see section 3.2 for details.

The slots are designated by six orbital elements. For a given altitude (and therefore semi-major axis), the range of $\Omega$ values is discretized by $3.75^{\circ}$ to produce 15 minute spacing in mean local time (MLT).[2] The eccentricity is chosen to be small $(e=.001$ in this work) though larger values are possible as well. Inclination is calculated by Equation 1.2 and the argument of perigee, $\omega$, is set to $90^{\circ}$ to create "frozen orbit" conditions that SSOs often utilize to minimize variations in altitude.[5] The final element is given by the phasing rule developed in Reference [2]:

$$
u=2 \Omega
$$

where $u$ is the argument of latitude $(u=\nu+\omega)$. Together, these elements designate the position of the primary slots; two secondary slots are defined relative to each primary slot at $+2.5^{\circ}$ and $+5^{\circ}$ in $u$. The discretization in $\Omega$ produces 96 
orbital planes at each altitude; with one primary and two secondary slots at each MLT there are 288 slots available per altitude. Figure 2.1 shows an example of the full constellation at a single altitude. Note that the slots do not move along the characteristic baseball seam appearance but instead follow the orbital paths indicated in grey. Actual implementation of the slot architecture would call for such a constellation at multiple altitudes; the optimal vertical stratification of the architecture is investigated in depth in Reference [8].

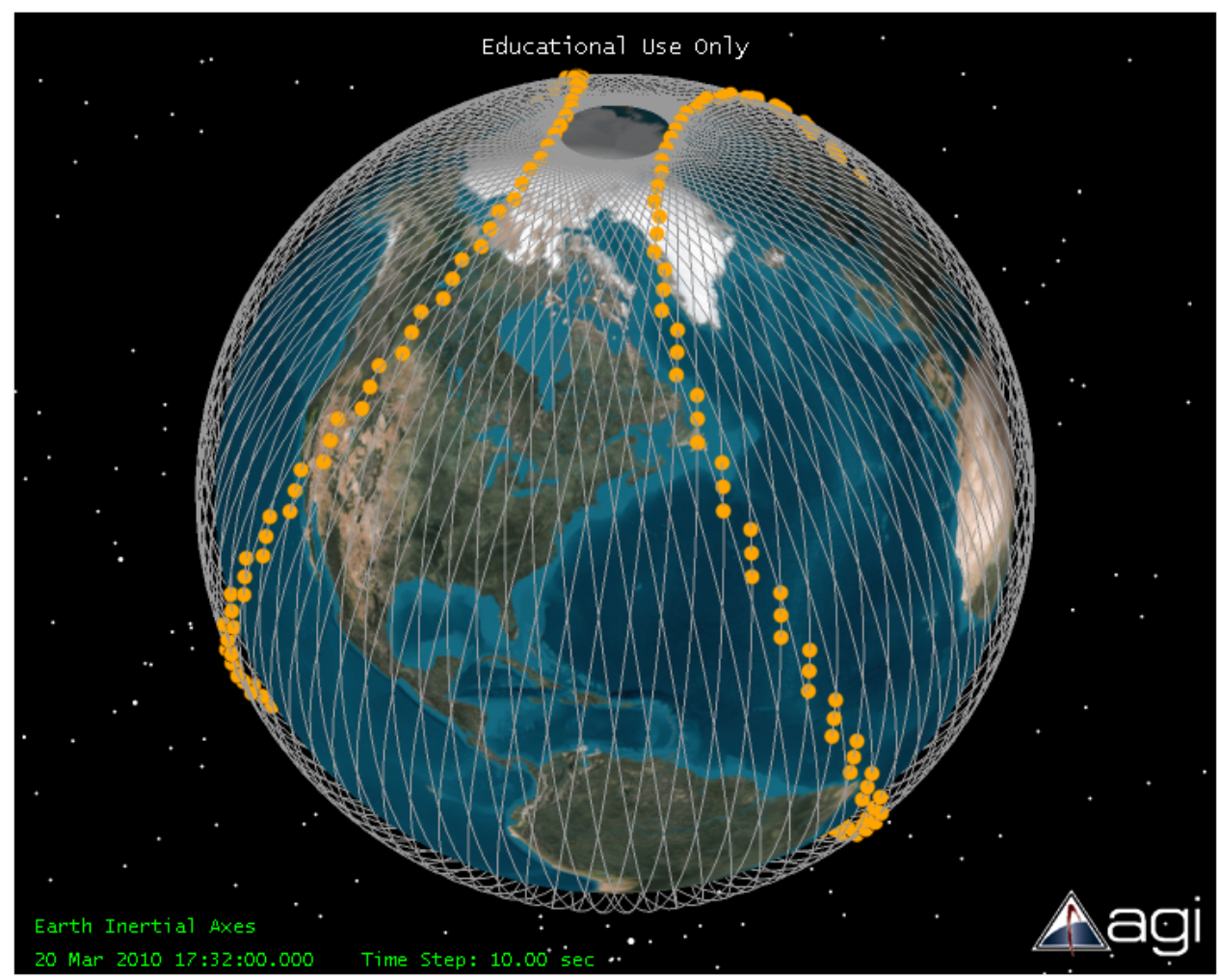

Figure 2.1: Architecture employed at a single altitude

At an individual level, this discretization of orbital elements may force satellites into less-than-ideal trajectories that result in performance losses. At a macroscopic level, however, the architecture can mitigate conjunctions, slow the growth of debris through reduction of collisions, and provide order and stability in this densely populated regime. 


\section{Chapter 3}

\section{Relative Motion Analysis}

The slots defined by the architecture are subject only to the point mass force of the Earth and the secular effects of $J_{2}$. In contrast, satellites experience a number of orbital perturbations including the effects of an oblate Earth, atmospheric drag, third-body gravitation, and solar radiation pressure. The satellite will move relative to its corresponding slot as a result. The objective of this section is to understand and characterize the effects of these perturbations on the motion of a satellite relative to its slot. Reference [7] performed a similar analysis at altitudes $500 \mathrm{~km}$ and $800 \mathrm{~km}$; this work extends that analysis to four additional altitudes as well as introduce several novel ideas.

Throughout this work, slot orbital elements are assumed to be Kozai-Izsak mean elements and are propagated using STK's J2Perturbation propagator. This propagator includes only the point mass effect and the secular drift in orbital elements resulting from $J_{2}$. All satellites are propagated using the High-Precision Orbital Propagator (HPOP) available in System Tool Kit (STK). HPOP is particularly useful because the various orbital perturbations can be activated in any combination; this allows for progression from simple scenarios (e.g., $J_{2}$ only) to 
more realistic cases that include atmospheric drag, Lunar and Solar gravity effects, and a high fidelity model of the Earth's gravity field. Before the satellites can be propagated using HPOP, the mean elements of the slot must be converted into their corresponding osculating elements. This conversion is performed via manipulation of STK's Astrogator tool.

\subsection{Method of Analysis}

In order to facilitate the relative motion analysis, a coordinate frame similar to Reference [7] is used. The origin of the coordinate system is placed at the slot and the motion is split into along-track, cross-track, and radial directions. The along-track dimension points in the direction of the velocity vector of the slot while the radial dimension points from the slot to the center of the Earth and the cross-track dimension completes the right-handed system. These quantities are calculated as

$$
\begin{aligned}
\hat{i} & =\frac{\vec{V}_{S L O T}}{\|\vec{V}\|} \\
\hat{k} & =\frac{-\vec{R}_{S L O T}}{\|\vec{R}\|} \\
\hat{j} & =\hat{k} \times \hat{i} \\
\Delta \vec{R} & =\vec{R}_{S A T}-\vec{R}_{S L O T} \\
\Delta \text { AlongTrack } & =\Delta \vec{R} \cdot \hat{i} \\
\Delta \text { CrossTrack } & =\Delta \vec{R} \cdot \hat{j} \\
\Delta \text { Radial } & =\left(\left\|\vec{R}_{S A T}\right\|-\left\|\vec{R}_{S L O T}\right\|\right) \hat{k}
\end{aligned}
$$


where $\vec{R}_{S A T}$ and $\vec{R}_{S L O T}$ are the Earth-centered inertial position vector of the satellite and slot, respectively, and $\hat{i}, \hat{j}$, and $\hat{k}$ are the unit vectors that define the coordinate system.

Although SSO is possible at a wide range of altitudes, the range $300-1000 \mathrm{~km}$ is examined because very nearly all active SSOs currently operate inside these altitudes. Review Figure 1.1 to see the current distribution of SSOs.

\subsection{Conformance Criterion}

One goal of conducting an analysis of the relative motion between satellites and their respective slots is to aid in developing a notion of what it means to comply with the proposed architecture. The criteria for conforming with the architecture must strike a balance between being stringent enough that the SSOs are well-separated and being sufficiently lax that slot maintenance maneuvers are not required so often as to impinge upon the satellite's mission.

The results of the relative motion analysis indicate that along-track drift occurs much more rapidly than in the cross-track and radial directions. After using STK's Advanced Conjunction Analysis tool to verify that the minimum horizontal separation created by the architecture is approximately $240 \mathrm{~km}$ irrespective of altitude,[2] the conformance criterion was set to be a $200 \mathrm{~km}$ drift in the along-track direction. Based on current operational practices, a LEO satellite "is considered to be safely separated whenever its $(25 \times 25 \times 2) \mathrm{km}$ [ellipsoidal] threat volume is clear of other space objects." [2] Thus, the conformance criterion allows complying satellites to remain separated in view of the current conjunction definition. 
The method of calculating the length of time a satellite is in conformance with the architecture varies depending whether atmospheric drag is taken into considering or not. For the scenarios in which only gravity is acting on the satellites, the conformance time is simply calculated as the time the satellite first reaches $200 \mathrm{~km}$ separation either ahead or behind its slot (results show that gravitational effects cause the satellites to lag behind their slots). This process has additional steps when drag is considered. Satellites experience drag differently based on their area, mass, and coefficient of drag. It is often useful to lump these quantities together as a property called the ballistic coefficient, typically defined as

$$
B C=\frac{m}{C_{D} A}
$$

where $C_{D}$ is the drag coefficient, $A$ is the satellite's area and $m$ is its mass. In order to get a worst-case estimate of the conformance times, a high drag satellite $\left(B C=50 \mathrm{~kg} / \mathrm{m}^{2}\right)$ is placed at a slot directly following a low drag satellite $\left(B C=200 \mathrm{~kg} / \mathrm{m}^{2}\right)$. Depending on altitude and solar conditions, a high drag satellite could outpace its slot due to a more rapid reduction in semi-major axis while a low drag satellite could fall behind its slot. In this case, the satellites could conjunct long before their individual along-track separation from their slots reaches $200 \mathrm{~km}$. Figure 3.1 portrays such a scenario graphically. If the satellites do not drift toward each other in this manner, the conformance time is then calculated as in the gravity-only scenarios and the high drag satellite will provide the worst-case estimate; low drag satellites can expect longer conformance times. 

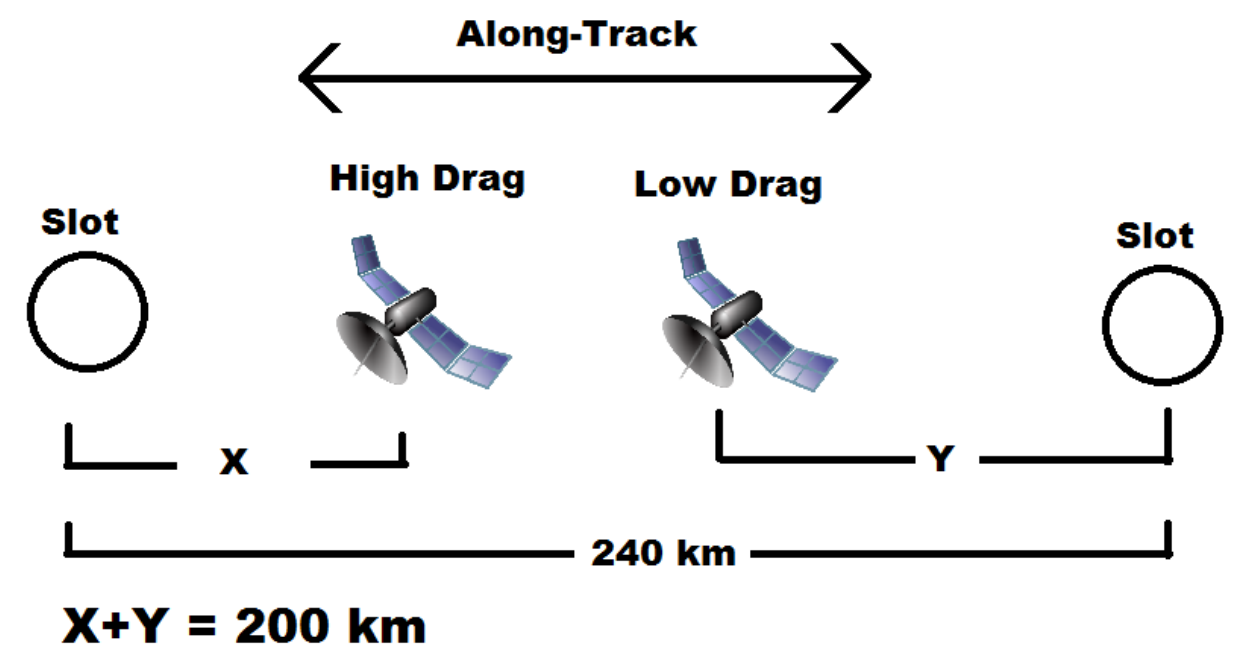

Figure 3.1: Representation of the conformance criterion for scenarios involving atmospheric drag

\subsection{Relative Motion Results}

\subsubsection{Gravity Only}

Initially only gravitational effects are considered. To start as simply as possible, the satellites are propagated with solely the effects of $J_{2}$ added to the two-body motion. This means the satellites experience both the secular and periodic gravitational effects while the slots are subject only to the secular effects. The short periodic effects on along-track, cross-track, and radial directions are shown in Figure 3.2 which displays the relative motion results over 24 hours. The same analysis over a six month period is shown in Figure 3.3; notably, there is secular drift in the along-track direction while motion in the cross-track and radial directions remains bounded.

In the next set of propagations, the satellites are subjected to higher order gravity effects. The gravity model used is STK's default model, the WGS84EGM96, the most up-to-date Earth Gravity Model. The model supports coef- 


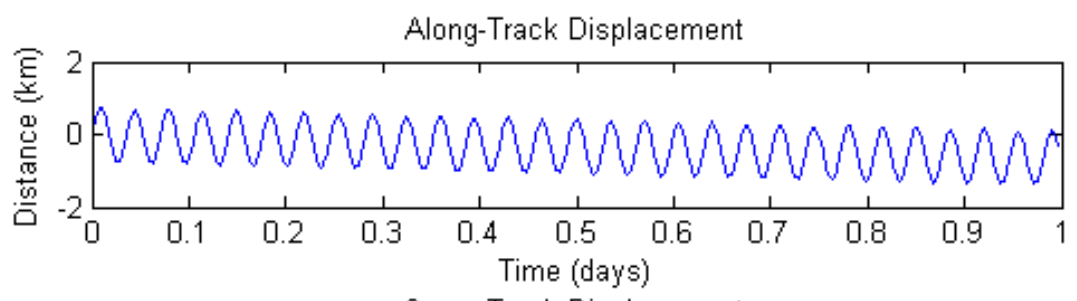

Cross-Track Displacement

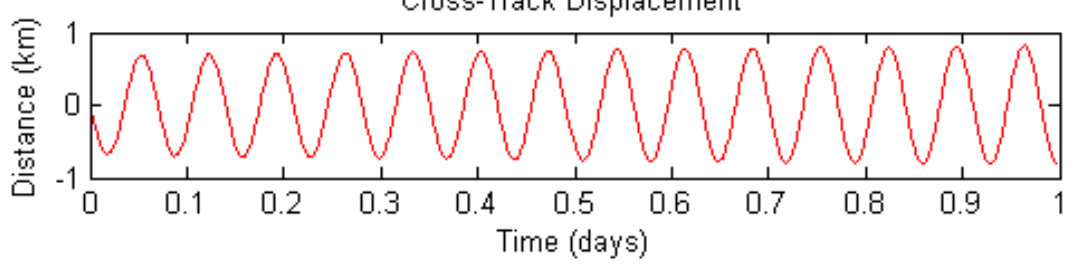

Radial Displacement

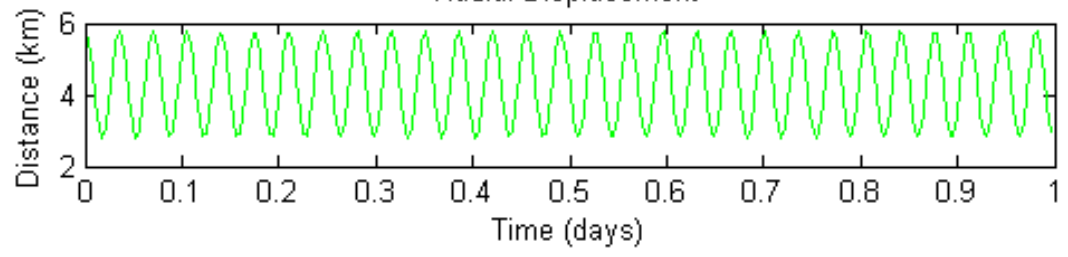

Figure 3.2: Motion of a satellite at $800 \mathrm{~km}$ altitude relative to its slot over one day propagated with the effects of $J_{2}$

ficients up to degree and order seventy. Two levels of fidelity are investigated: the STK default of 21x21 and the maximum values of 70x70, a standard in highprecision orbital propagation.

The short term results of the $70 x 70$ analysis are shown in Figure 3.4. The results are similar to the $J_{2}$ only case, though the higher order effects are clearly visible in the along-track and cross-track directions. The inclusion of higher-order terms introduce long periodic effects in along-track and radial dimensions not seen in the $J_{2}$ analysis; this behavior can be seen in Figure 3.5. The $21 \mathrm{x} 21$ gravity results (not shown) show no appreciable difference to the 70x70 model. Both the long and short term results agree strongly with those presented in Reference [7]. The growing cross-track amplitude seen in Figure 3.5 can be traced to a slight secular drift in the satellite's orbital inclination and RAAN relative to its slot. 


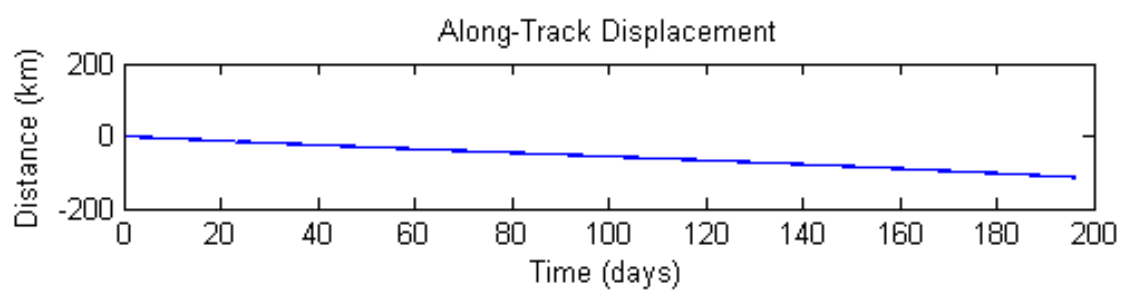

Cross-Track Displacement

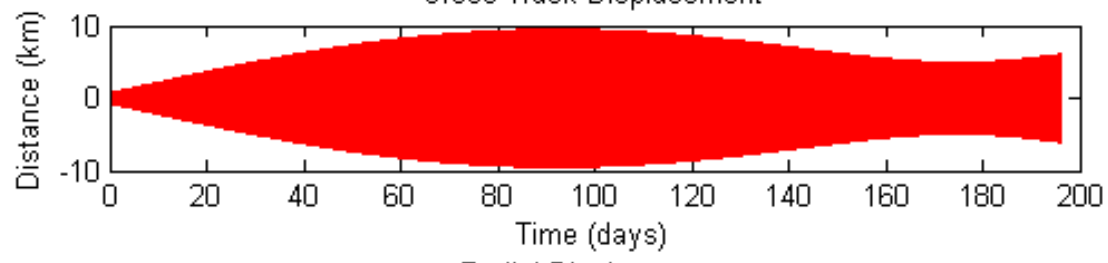

Radial Displacement

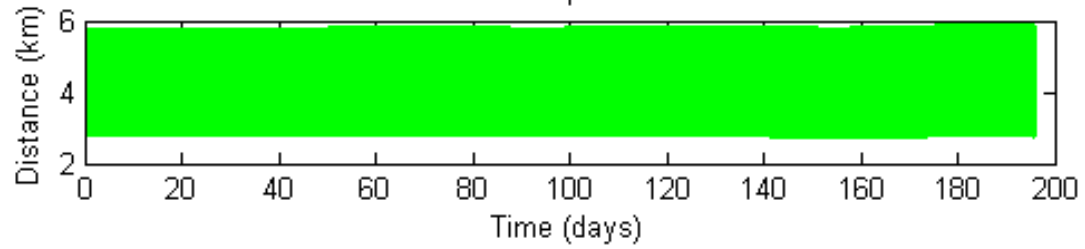

Figure 3.3: Motion of a satellite at $800 \mathrm{~km}$ altitude relative to its slot over six months propagated with the effects of $J_{2}$

The conformance time results for the $J_{2}$ only case are quite high due to the very similar dynamics between the slot and satellite. As seen in Figure 3.6, the times linearly increase with altitude from about 230 days at $300 \mathrm{~km}$ altitude up to nearly 350 days at $1000 \mathrm{~km}$. Introducing higher-order gravity effects resulted in a non-monotonic behavior though in a coarse sense the conformance times do tend to increase with altitude as they did in the $J_{2}$ only case. Across all altitudes, the additional gravity effects result in a reduction in conformance time. Although different in magnitude, the general trend is similar for both the $21 \times 21$ and $70 \times 70$ propagations. 


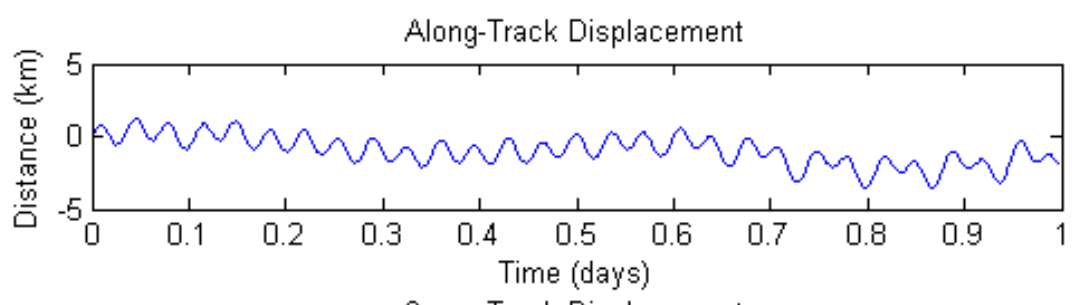

Cross-Track Displacement

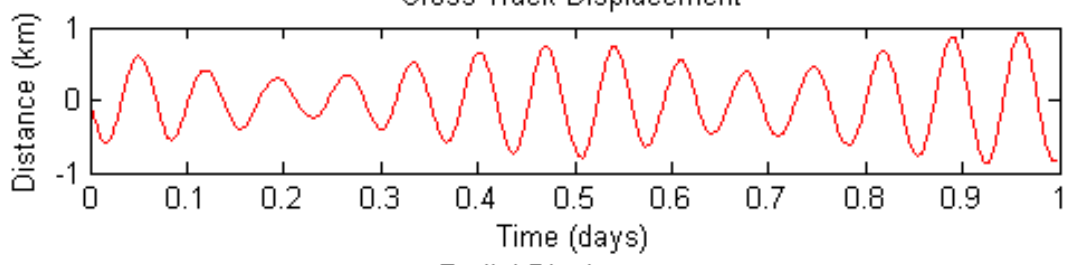

Radial Displacement

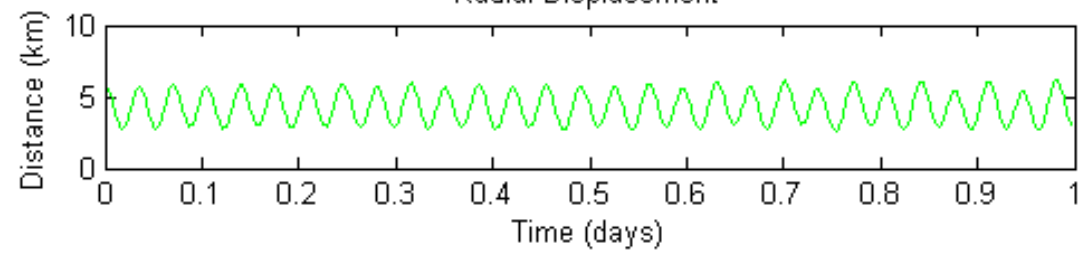

Figure 3.4: Motion of a satellite at $800 \mathrm{~km}$ altitude relative to its slot over one day propagated with the $70 \times 70$ gravity field

\subsubsection{Gravity and Atmospheric Drag}

The satellites are now propagated using the $70 x 70$ gravity model and atmospheric drag. At each altitude, two satellites with two different ballistic coefficients are propagated as mentioned in Section 3.2; this is done for three different solar cycle conditions. The solar conditions (minimum, average, and maximum) correspond to F10.7 values of 69, 118.7, and 189 solar flux units,[9] respectively, where $1 \mathrm{SFU}=10^{-22} \mathrm{Wm}^{-2} \mathrm{~Hz}^{-1}$. Atmospheric drag has a particularly strong effect on satellites at low altitudes; this effect is most pronounced during a solar maximum. Specifically, drag tends to cause a reduction in semi-major axis, thereby increasing the satellite's orbital velocity. The result is rapid along-track separation from the slot and a concomitant decrease in conformance time.

The results of a satellite propagated with a ballistic coefficient of 50 and maxi- 

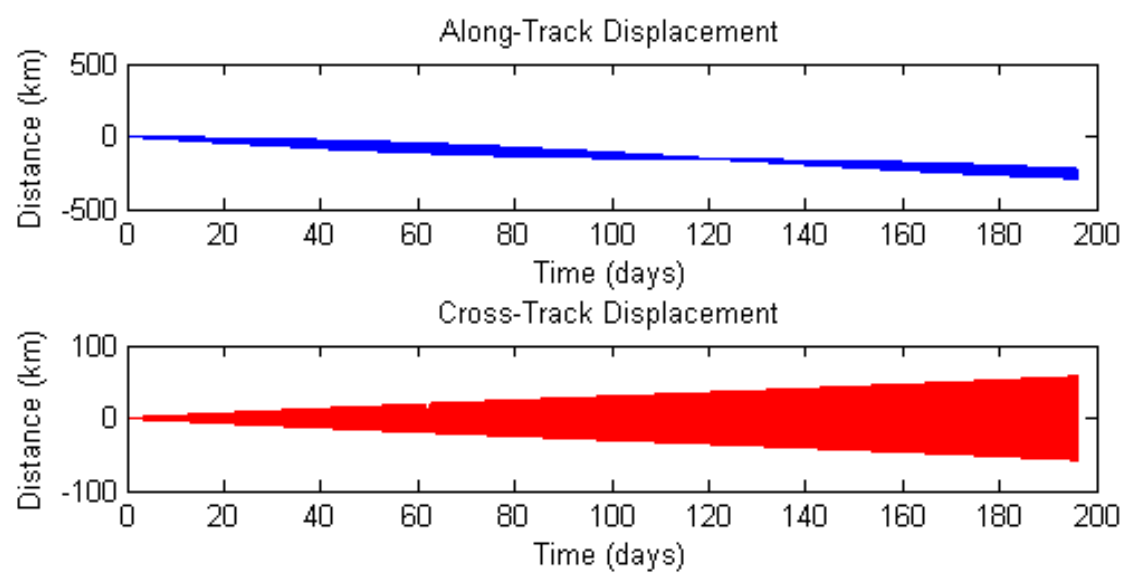

Radial Displacement

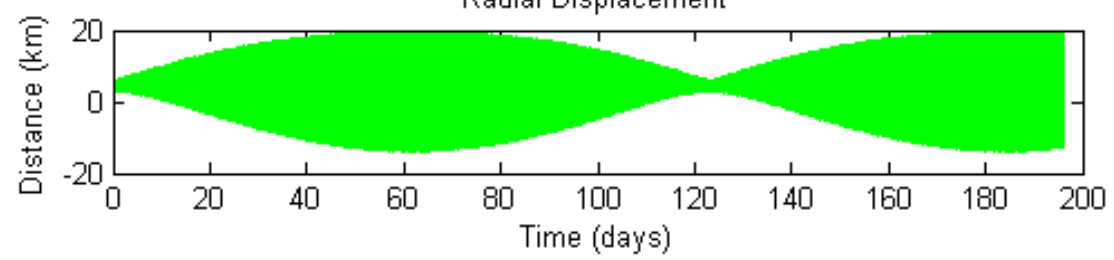

Figure 3.5: Motion of a satellite at $800 \mathrm{~km}$ altitude relative to its slot over six months propagated with the $70 \times 70$ gravity field

mal solar conditions is shown in Figure 3.7. The cross-track and radial dimensions are not significantly different from their gravity only counterparts. The results are displayed over a ten day period because the results are nearly indistinguishable from their gravity-only counterparts over a single day. For approximately four days, the gravitational effects counteract the effects of atmospheric drag. Afterward, the satellite very quickly leads the slot in the along-track direction.

The along-track motion of both a high drag and a low drag satellite during maximal solar conditions is shown in Figure 3.8. Mean lines are calculated using an exponentially weighted moving average to filter out the short periodic effects. These mean values are used to calculate the conformance time shown in red. The high drag satellite shows a much quicker separation than the low drag satellite as one would expect. Because both satellites move ahead of their slots, the 


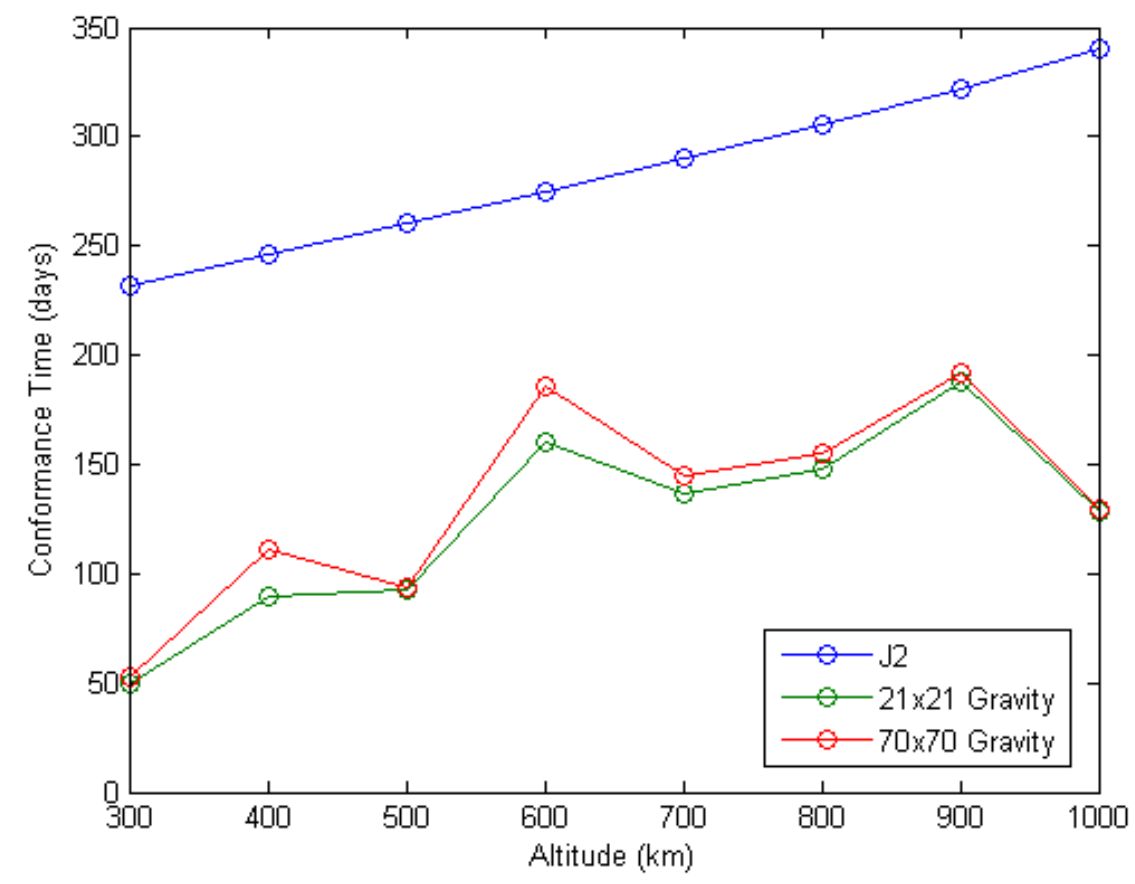

Figure 3.6: Conformance times for gravity-only scenarios

conformance time is simply the period in which the absolute along-track drift of both satellites is less than $200 \mathrm{~km}$; in this case, the high drag satellite reaches $200 \mathrm{~km}$ first after approximately 33 days. Figure 3.9 shows a case in which atmospheric drag causes a satellite with a low ballistic coefficient (i.e. high drag) to drift ahead of its slot while a satellite with a high ballistic coefficient falls behind its slot. In this case, as explained in Section 3.2, the conformance time is calculated as the period in which the sum of the absolute along-track drift of both satellites reaches $200 \mathrm{~km}$ which occurs after approximately 115 days. The conformance time results are calculated by performing this analysis across all altitudes and for the other solar cycle conditions.

The conformance time results are shown in Figure 3.10. As stated, the conformance times are reduced, relative to the gravity-only case, across all altitudes for all solar cycle conditions. The conformance times are lowest during maxi- 

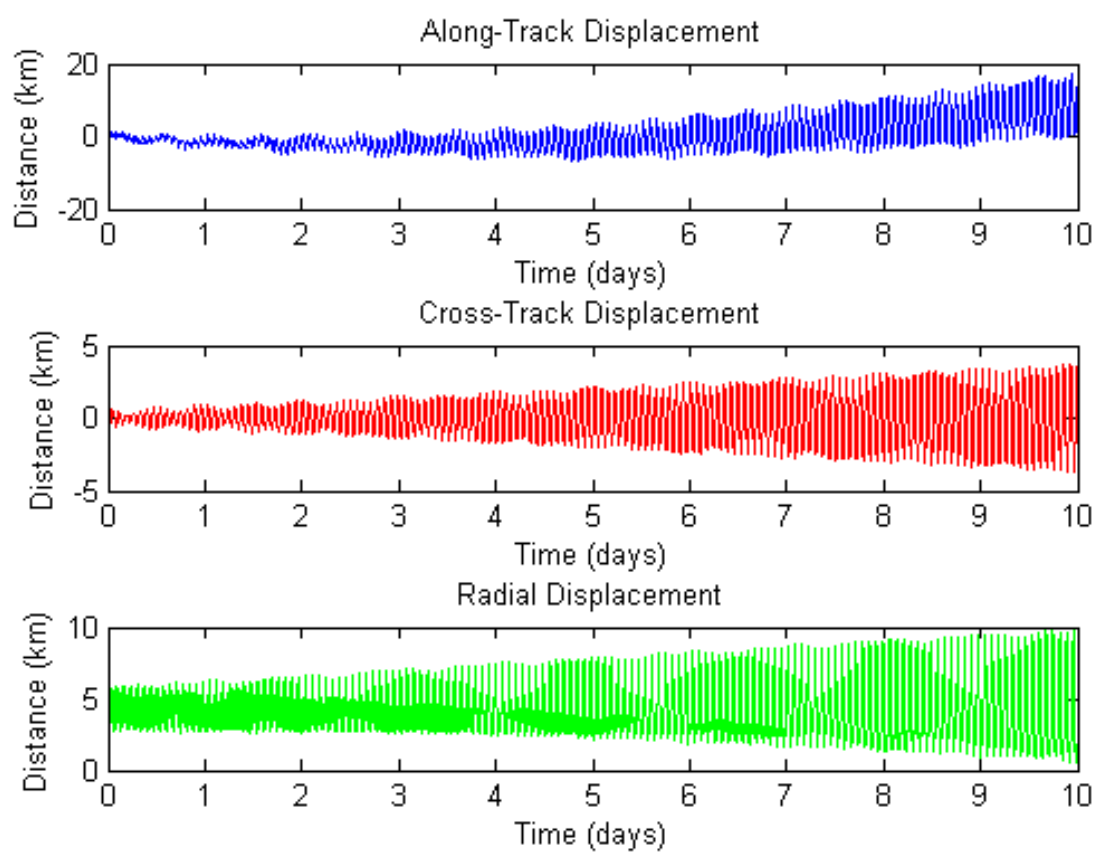

Figure 3.7: Motion of a satellite at $800 \mathrm{~km}$ altitude relative to its slot over ten days propagated with the $70 \times 70$ gravity field and atmospheric drag

mal solar conditions and highest during minimal solar conditions as one would expect. The non-monotonicity of the $70 x 70$ gravity model is smoothed by the dominant effects of atmospheric drag at low altitudes. At higher altitudes, the effects of drag diminish significantly and again the gravitational effects (as well as SRP) would become significant. At the lowest altitudes, those less than 500 $\mathrm{km}$, the effects of drag reduce the conformance times to the order of hours and days; for satellites that do not already perform maintenance maneuvers with such frequency, requiring that they maintain in conformance with their assigned slot could prove very costly in terms of propellant as well as detrimental to their mission. It is important to recall, however, that the values presented are worst-case scenarios in which a high drag satellite resides in a slot immediately behind a low drag satellite. 


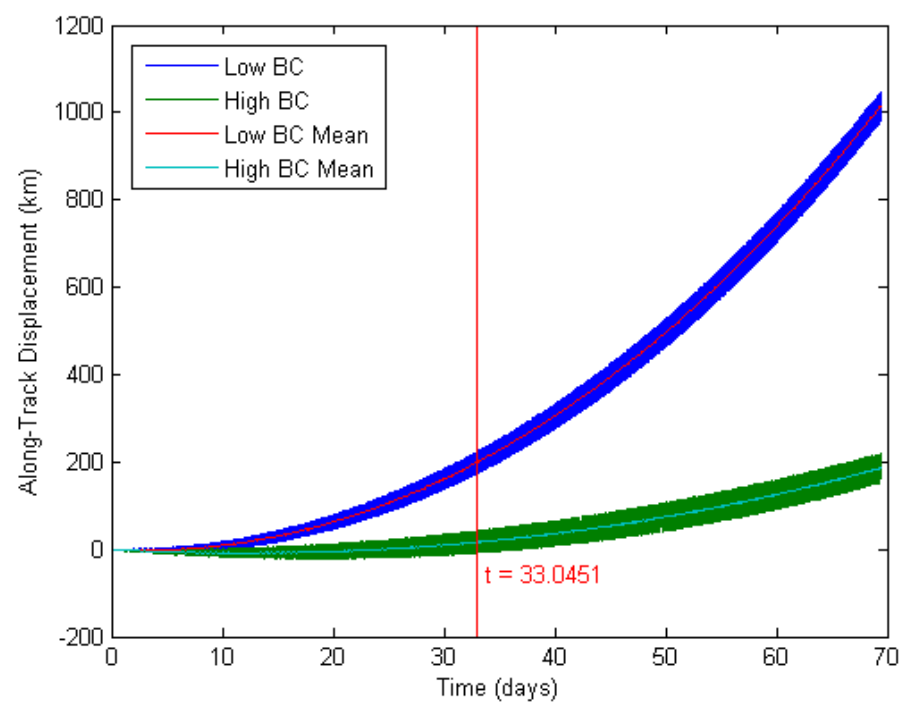

Figure 3.8: Along-track separation of a satellite at $800 \mathrm{~km}$ during solar maximum conditions

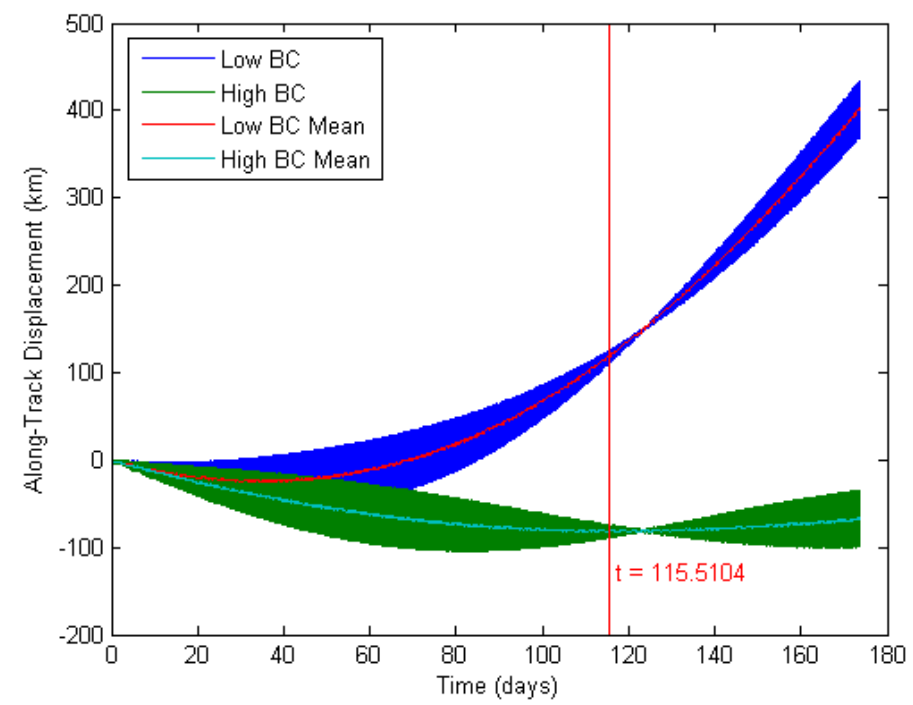

Figure 3.9: Along-track separation of a satellite at $800 \mathrm{~km}$ during solar minimum conditions 


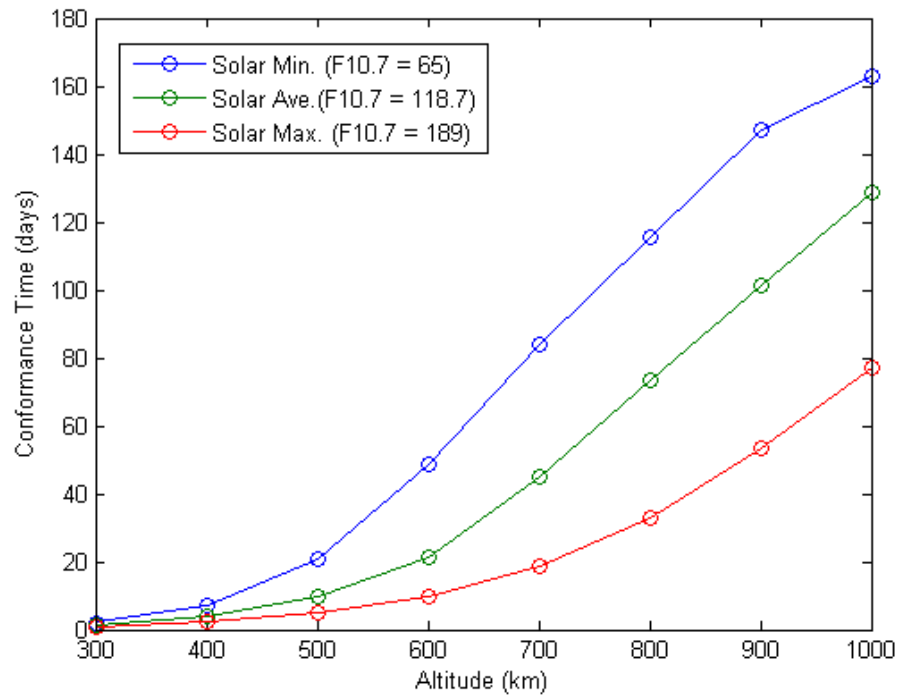

Figure 3.10: Worst-case estimate of conformance time vs altitude for satellites propagated with 70x70 gravity field model and atmospheric drag 


\section{Chapter 4}

\section{Orbital Debris Environment}

\section{Model}

This chapter marks a shift in focus. The previous chapter was an in-depth look at the relative motion of individual satellites and their slots over time. The focus of this chapter is the Earth's orbital debris environment as a whole, and how the proposed sun-synchronous orbit slot architecture will affect it. High fidelity evolutionary debris environment models like NASA's LEO-to-GEO Environment Debris model (LEGEND) conduct three-dimensional object-by-object simulations and typically predict collisional behavior based on the NASA Standard Breakup Model. LEGEND includes the perturbing effects of $J_{2}, J_{3}$, and $J_{4}$, solar-lunar gravitational effects, atmospheric drag, solar radiation pressure, and the effects of Earth's shadow. The model features uncertainties and thus results are typically an average of upwards of 100 Monte Carlo simulations.[10]

A number of attempts have been made at capturing the complexities of models such as LEGEND by using ordinary differential equations (ODE) to model the 
orbital debris environment as a set of sources (e.g. future launches, collisions, explosions, etc.) and sinks (e.g. atmospheric decay and end-of-life deorbiting).[1113] Notable among these is the model developed by Bradley and Wein [11]. The model is comprised of five coupled ODEs that track the number of rocket bodies, three types of spacecraft, and four forms of fragments over time. One way in which the model differentiates itself from others is the introduction of a nonuniformity factor to capture the increased collision rates near the Earth's poles. This is useful when dealing with SSOs due to their clustering near the poles; capturing the spatial density of the environment is essential to accurately modeling the effects of the slot architecture. With some modification, the model lends itself well to the task of analyzing the benefits of implementing the architecture. Hereafter, any reference to "the model" will refer to the framework of Reference [11] unless stated otherwise.

Even the highest fidelity models necessarily introduce assumptions, the model of Reference [11] included. The model develops only collisional interactions between satellites; rocket body explosions are neglected. The original framework simulated the region between 900 and $1000 \mathrm{~km}$ altitude and ignored the effects of collisions above or below this region. All intacts, i.e., rocket bodies and spacecraft, are assumed to be in circular orbits and therefore spend their entire period within the region. The nonuniformity factor reflects the spatial distribution of the current environment; this distribution is assumed constant throughout integration of the model.

The model tracks the number of rocket bodies, active spacecraft with deorbit capability, active spacecraft without deorbit capability, inactive spacecraft, and four types of fragments over time. Rocket bodies and the the three types of spacecraft are collectively referred to as intacts. Fragments are assumed to inherit 
the characteristics of their satellite of origin, either a spacecraft or rocket body. Fragments are additionally deemed hazardous or benign based on their ability to destroy an intact in a hypervelocity collision. Because the geometry and velocity of a collision cannot be known beforehand, fragments are not entirely hazardous or benign. Instead, their properties determine the probability with which it can destroy an intact. "A particular fragment from source $\tau \in\{R, S\}$, therefore, increases the effective number of $F_{\tau}^{h}$ and $F_{\tau}^{b}$ by quantities that sum to one."[11]

Much of the complexity of the model lies in calculating the ODE coefficients. These parameters are the weighted expectations over the same empirically derived probability density functions that are used in object-by-object models like LEGEND.

\subsection{Model Additions and Modifications}

A few select changes to the model were required in order to better suit the needs of this work.

\subsubsection{Extension to Additional Altitudes}

The original model was limited to the shell of interest (SOI) between the altitudes 900 and $1000 \mathrm{~km}$. The region of interest in this work extends from $500 \mathrm{~km}$ altitude up to $1000 \mathrm{~km}$. It was thus necessary to determine the model parameters for other SOI. Monte Carlo integration is used to calculate the expected value of the model parameters weighted by fraction of time an object spends in the SOI and its ability to cause a catastrophic collision with an intact. The entire region cannot be accurately analyzed as a single SOI using this model because the model 
parameters are assumed constant through an SOI. That is, it is assumed that the decay rate, for example, of an object at $950 \mathrm{~km}$ altitude is sufficiently similar for objects at the extrema of the SOI, i.e., at 900 and $1000 \mathrm{~km}$ altitude. To continue the example, the decay rate of an object at $500 \mathrm{~km}$ is approximately two orders of magnitude faster than that of an object with the same area-to-mass ratio at $1000 \mathrm{~km}$.[13] The region is therefore discretized into the following altitude pairs: $500-550 \mathrm{~km}, 550-600 \mathrm{~km}, 600-700 \mathrm{~km}, 700-800 \mathrm{~km}, 800-900 \mathrm{~km}$, and the original SOI 900-1000 km. A sensitivity analysis is performed to determine if the spatial discretization is sufficiently small at the lower end to capture the effects of decay of fragments into and out of the SOI.

\subsubsection{Decay into the Shell of Interest}

Fragments above the original 900 to $1000 \mathrm{~km}$ SOI will decay very slowly into the SOI due to the trace amounts of atmosphere still present. The original model set the arrival rate of new fragments to zero because of this slow decay rate. At lower altitudes, however, the decay rate is much faster and thus objects will enter the SOI at a non-negligible rate. To capture this effect, the term $\lambda_{F_{\tau}^{\kappa}}$ is introduced into Equation 4.5. This parameter is estimated using the decay rates

given in Reference [13], the weighted expected area-to-mass ratio of each type of fragment from the region above the SOI, and the initial number of fragments from the shell above the SOI. This parameter is held constant despite the reality that it will change as the debris population changes. 


\subsubsection{Parameter Growth}

Many of the model parameters are assumed constant through integration of the ODEs. For some of the parameters, such as the average lifetime of a spacecraft in a given SOI, this assumption is perfectly valid. For others, however, a more realistic rate of change can be introduced into the model. In particular, the annual number of spacecraft launched into LEO is likely to grow due to the emergence of new technologies and falling costs associated with launches. A launch rate growth parameter has been added to the model and the number of launches per year is now modeled as an additional ODE, see Equation 4.6. The behavior of the original model can be reproduced by setting the growth rate, $\eta=0$. The fraction of future spacecraft that will have deorbit capability is also likely to grow in the

future. Equation 4.7 models this growth; setting $\theta_{\max }=\theta_{d}(0)$ will reproduce the original, fixed-value model. 


\subsection{The Environment Model}

The altered model is given by the following set of ODEs:

$$
\begin{aligned}
\dot{R}(t) & =\lambda_{R}-\sum_{\alpha \in U^{h}} \beta_{R \alpha} R(t) \alpha(t)-\mu_{R} R(t) \\
\dot{S}_{n}^{o}(t) & =\left(1-\theta_{d}(t)\right) \lambda(t)-\sum_{\alpha \in U^{h}} \beta_{S \alpha} S_{n}^{o}(t) \alpha(t)-\mu_{o} S_{n}^{o}(t) \\
\dot{S}_{n}(t) & =\mu_{o} S_{n}^{o}(t)-\sum_{\alpha \in U^{h}} \beta_{S \alpha} S_{n}(t) \alpha(t)-\mu_{n} S_{n}(t) \\
\dot{S}_{d}(t) & =\theta_{d}(t) \lambda(t)-\sum_{\alpha \in U^{h}} \beta_{S \alpha} S_{d}(t) \alpha(t)-\mu_{o} S_{d}(t) \\
\dot{F}_{\tau}^{\kappa}(t) & =\lambda_{F_{\tau}^{\kappa}}+\frac{1}{2} \sum_{\alpha \in U} \sum_{\gamma \in U} \delta_{\alpha \gamma}^{\tau \kappa} \alpha(t) \gamma(t)-\mu_{F_{\tau}^{\kappa}} F_{\tau}^{\kappa}(t) \\
\dot{\lambda}(t) & =\eta \lambda(0) \\
\dot{\theta}_{d}(t) & =\frac{\theta_{\max }-\theta_{d}(0)}{a} e^{-t / a}
\end{aligned}
$$

where $R(t)$ is the number of rocket bodies, $\lambda_{R}$ is the annual rate at which rocket bodies are inserted into the SOI, $U^{h} \equiv\left\{S, R, F_{S}^{h}, F_{R}^{h}\right\}$ is the set of satellite types hazardous to intacts (intacts refers to all rocket bodies and spacecraft whether active or inactive), $\beta_{\alpha \gamma}$ is the collision rate parameter between satellites of type $\alpha, \gamma \in U \equiv\left\{S, R, F_{S}^{h}, F_{S}^{b}, F_{R}^{h}, F_{R}^{b}\right\}, \mu_{R}$ is rate at which rocket bodies deorbit naturally, $S_{n}^{o}(t)$ is the number of active spacecraft without deorbit capability, $\theta_{d}(t)$ is the fraction of future spacecraft that have deorbit capability, $\lambda(t)$ is the annual number of spacecraft inserted into the SOI, $\mu_{o}$ is the inverse of average operational lifetime, $S_{n}(t)$ is the number of inactive spacecraft, $\mu_{n}$ is the rate at which satellites naturally leave the SOI, $S_{d}(t)$ is the number of active satellites with deorbit capability, $F_{\tau}^{\kappa}(t)$ is the number of fragments of type $\kappa \in\{h, b\}$ and $\tau \in U^{I} \equiv\{S, R\}, \lambda_{F_{\tau}^{\kappa}(t)}$ is the rate at which fragments enter the SOI, $\frac{\delta_{\alpha \gamma}^{\tau \kappa}}{\beta_{\alpha \gamma}}$ is 
roughly the number of fragments of a certain type generated by a certain type of collision, $\eta$ is the launch rate escalation factor, $\theta_{\max }$ is the maximum post-mission deorbit compliance rate, and $a$ is simply a value that determines over what time scale the change from $\theta_{d}(0)$ to $\theta_{\max }$ occurs.

The goal of the model is to use it to coarsely estimate the effect of the slot architecture on conjunctions, actual collisions, and the overall orbital debris environment. The model is first used to establish a baseline to serve as a point of reference. Then, the model is adjusted to account for the implementation of the slot architecture; the effects of the architecture are quantified by comparing the results.

\subsubsection{Model Validation}

In order to ensure that the model works as intended, the results of a 900 to $1000 \mathrm{~km}$ SOI run are compared against those of Reference [11]. Keeping in mind that the coefficients are the result of a large number of Monte Carlo integrations, the results will statistically never be identical. Figures 4.1 and 4.2 compare several quantities tracked by the model. Results of the original paper are indicated by the author A.M. Bradley's initials while the results produced by the recreated model are designated by my own initials, "CDN". The results of this data generation run differ from the original model by no more than $0.53 \%$ in the total number of intacts and $0.77 \%$ in effective number of fragments, though other data generations produced results that differ by as much as $5 \%$ due to variations in the Monte Carlo integrations. Nevertheless, the excellent agreement indicates that the recreated model accurately reproduces the behavior of original model. 


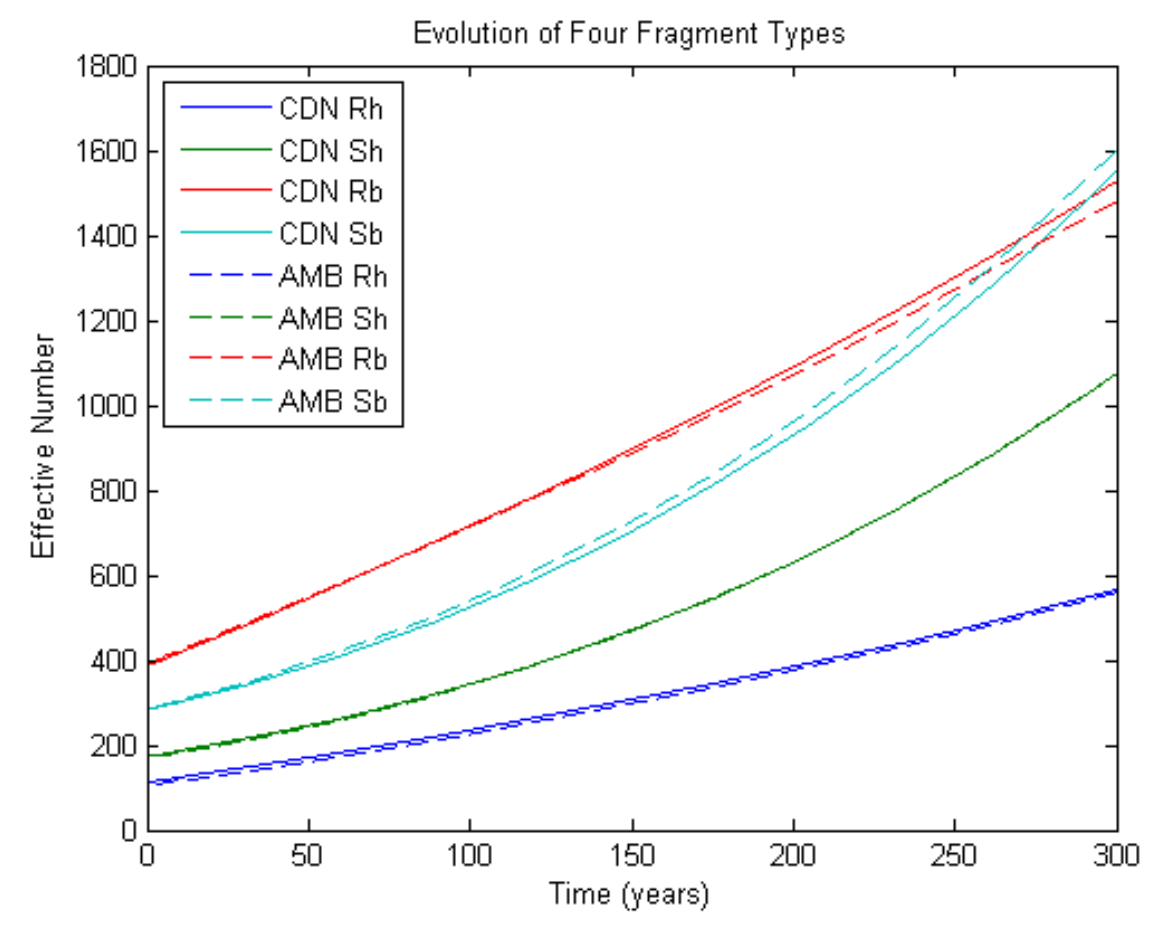

Figure 4.1: Fragment growth over time
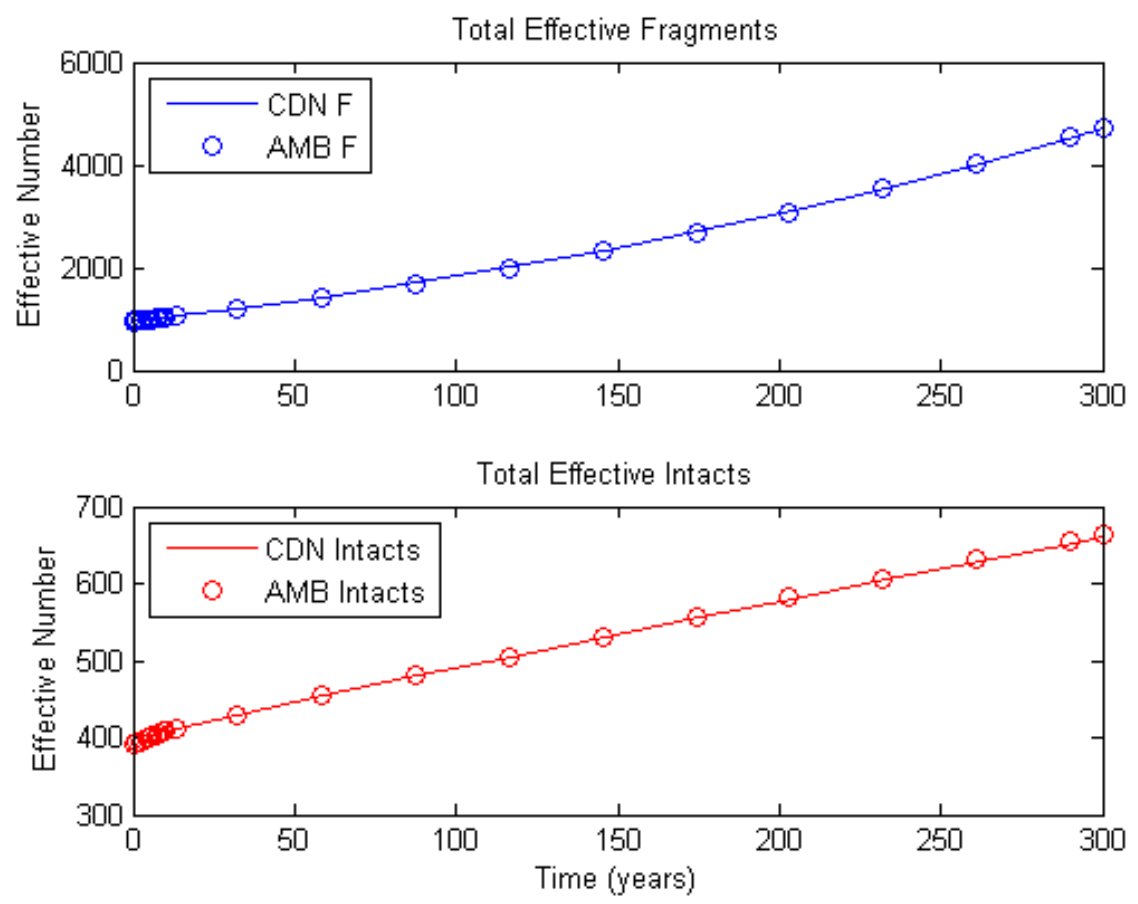

Figure 4.2: Total fragments and intacts over time 


\subsubsection{SOI Discretization Sensitivity Analysis}

A brief sensitivity analysis was conducted in order to determine if splitting the lowest altitudes into SOI of $50 \mathrm{~km}$ sufficiently captured the increased sensitivity to decay into and out of the shell. The lowest SOI from 500 to $550 \mathrm{~km}$ mean altitude is discretized into five evenly distributed $10 \mathrm{~km}$ shells and the sum of the results is compared to the result of the single shell. In order to hone in on the increased effects of residual atmosphere, the model is run with no future launches, full compliance with rocket body deorbiting, and all initial spacecraft are nonoperational and are therefore incapable of deorbiting.

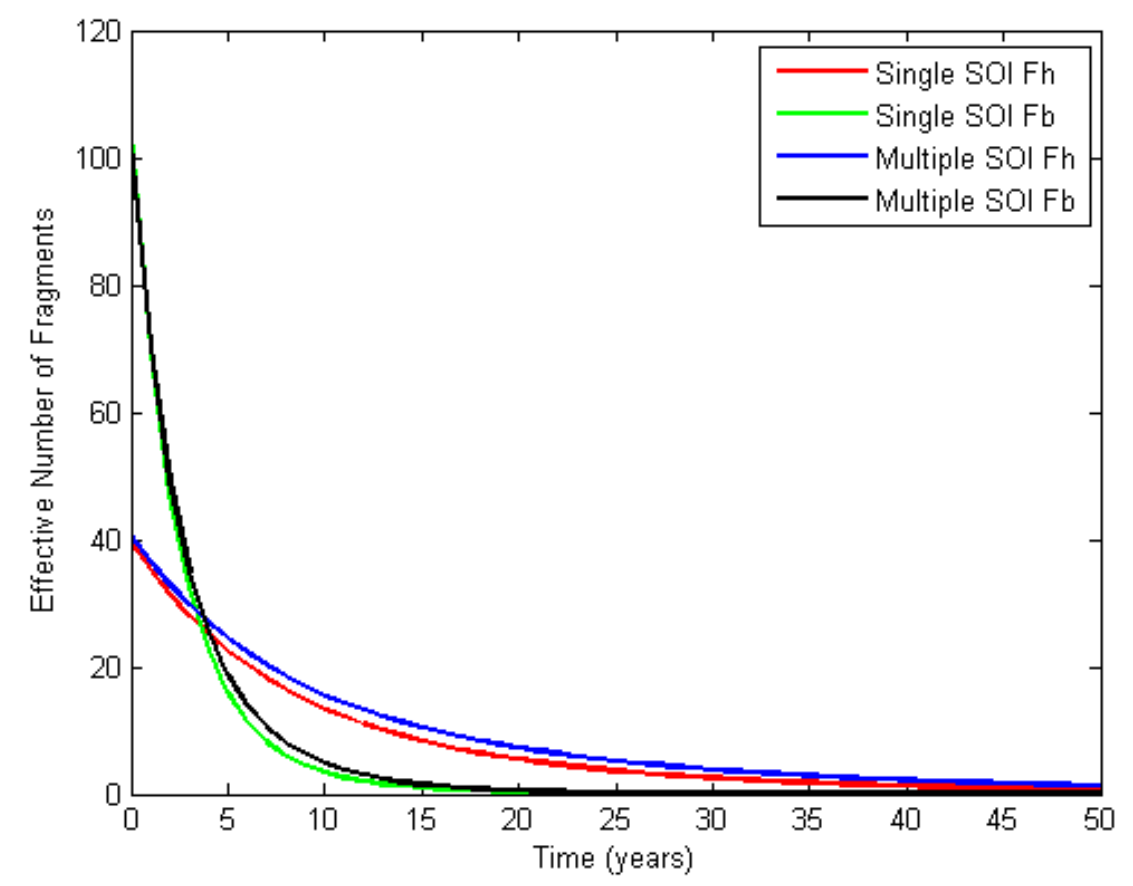

Figure 4.3: Comparison of the evolution of hazardous and benign fragments for two different spatial discretizations

The decay rate into an SOI was added solely for fragments, not for spacecraft or rocket bodies. Therefore, the evolution of the fragment environment is examined to determine if the spatial discretization is sufficient. Figure 4.3 shows 
the results of a single SOI versus multiple SOI. The results differ by no more than $15 \%$ and typically differ by less than $10 \%$ throughout integration of the model. Unlike the fragment evolution of the entire 500 to $1000 \mathrm{~km}$ region shown in Chapter 5, the number of fragments decays toward zero in Figure 4.3. This is because the rate at which fragments leave the SOI is faster than the rate at which they enter due to the difference in altitude. 


\section{Chapter 5}

\section{Numerical Results}

This chapter details the results of the environment model under various conditions.

\subsection{Baseline Scenario}

The baseline case is meant to capture a business-as-usual scenario by using recent history and the current environment to inform choices for key parameters such as average operational lifetime, spatial distribution of satellites, and the number of launches per year. The following section details these choices and calculations.

\subsubsection{Parameter Estimation}

The model has a number of input parameters that must be tailored to the study at hand. The initial conditions are calculated using the full catalog of two-line element (TLE) sets available from Space Track.[4] The TLEs are sepa- 
rated into rocket bodies, indicated by "R/B"; fragments, indicated by "DEB", "COOLANT", "FUEL", or "NEEDLES"; and spacecraft. Spacecraft are further split into active and inactive; a spacecraft is considered active if the NORAD catalog number from its TLE can be cross-listed against the Union of Concerned Scientists' (UCS) database of active spacecraft.[3] Using this process, 923 of the reported 1046 active spacecraft are matched. Of these 923, 436 are in LEO and 193 are found to be with $\pm 5 \%$ of SSO condition. This places $(193 / 436 \times 100=)$ $44.3 \%$ of active LEO satellites in SSO which echoes the result found in Reference [2]. Each object in the catalog is propagated for one full period and increases the initial number of its respective category by the fraction of time it spends in the SOI. For example, a piece of Fengyun-1C debris that spends $25 \%$ of its period between the upper and lower altitude of an SOI increases $F_{S}(0)$ for the SOI by 0.25. This is referred to as the effective number.

Table 5.1 lists values for average operational lifetime, number of spacecraft insertions per year, and percentage of future spacecraft that will comply with NASA's 25-year post-mission deorbit guideline. Average operational lifetime values are based on historical averages coupled with the fact that lifetimes tend to be higher at higher altitudes where the degrading effects of atmospheric drag have diminished. The number of spacecraft insertions is based the historical number of launches into LEO each year. In previous years, the total number of launches was approximately 75 per year, with roughly 40 of these being LEO satellites.[14, 15] The number of launches into each SOI is allocated based on the fraction of the current environment in each SOI, e.g., if $10 \%$ of the current population resides in the region between 800 and $900 \mathrm{~km}$ altitude and the total number of LEO launches per year is 40, then the number of insertions per year into the SOI is set to 4 . The results of these calculations are displayed in Table 5.1. 
The percentage of spacecraft that comply with the post-mission deorbit guideline set forth by NASA is difficult to estimate. In Reference [16] NASA states that for satellites below $1400 \mathrm{~km}$ altitude, i.e. all of the satellites considered herein, "the most energy-efficient means of disposal of space structures is via maneuver to an orbit from which natural decay will occur within 25 years" [16] which typically occurs below $600 \mathrm{~km}$ altitude.[11] The compliance rate for the two lowest SOI, both of which are beneath $600 \mathrm{~km}$ altitude, is thus set to $100 \%$. The rates for other SOI are more difficult to estimate because it is not well known what fraction of LEO satellites have maneuverability; furthermore, those that do have such capability are not guaranteed to comply. In order to estimate the fraction of satellites with maneuverability, the UCS database was again utilized. Considering only U.S. satellites, the database was sorted by operator and by purpose. The satellites known to have no propulsive capability, such as nanosatellites operated by universities, are removed from the list. It is assumed that most U.S. military, NASA, and NOAA satellites are maneuverable. Finally, satellites whose purpose is reconnaissance, Earth observation, surveillance, or scientific are likely to have propulsive capability. Thus, an estimated 150 of U.S. operated LEO satellites have some form of maneuver capability, totaling approximately 60\%. Nicholas Johnson and Eugene Stansbery similarly estimate 700 of the 1100 (=64\%) operational satellites are maneuverable in some capacity (private communication, May 2012). As a conservative estimate and accounting for the possibility that a smaller fraction of non-U.S LEO satellites have maneuver capability, it is assumed that $50 \%$ of all LEO satellites have such capability. The compliance rate for SOI above $600 \mathrm{~km}$ is therefore set to the relatively low value of $50 \%$.

The model is also capable of allowing active satellites to avoid collisions; a key input is the fraction of collision avoidance maneuvers that are successful. The 
Joint Space Operations Center (JSpOC) tracks active satellites and issues Conjunction Support Messages in the event of a close approach. However, a study performed by Intelsat found that these conjunction warnings have nearly a 50\% false positive in which a warning was issued despite there being no risk of collision, and $50 \%$ false negative in which no warning was issued for an actual close approach.[17] Some conjunctions will therefore result in collisions. Furthermore, only active satellites with maneuver capability are able to avoid collisions. The model is run with both extreme values, i.e., 0\% (active satellites avoid all collisions) and 100\% (no collision avoidance), as well as an intermediate success rate of $40 \%$ which is considered to be the most realistic case.

See Reference [11] for details in computing the parameters regarding collision rates, debris generation, decay rates, and spatial nonuniformity. The collision rate parameters govern how frequently collisions occur between any two types of objects. The debris generation parameters determine how many and of what type new fragments are for a given collision. The decay rates determine the rate at which objects decay out of a given SOI, and the nonuniformity factors capture the distribution of objects in the current environment. All of these parameters are fixed for each SOI.

Table 5.1: Key model parameters as they vary with shell of interest

\begin{tabular}{|c|c|c|c|}
\hline $\begin{array}{c}\text { SOI } \\
(\mathrm{km})\end{array}$ & $\begin{array}{c}\text { Average Operational } \\
\text { Lifetime (years) }\end{array}$ & $\begin{array}{c}\text { Spacecraft Insertions } \\
\text { Per Year }\end{array}$ & $\begin{array}{c}\text { Post-Mission } \\
\text { Deorbit Compliance }\end{array}$ \\
\hline $500-550$ & 3 & 2 & $100 \%$ \\
\hline $550-600$ & 3 & 2 & $100 \%$ \\
\hline $600-700$ & 3 & 7 & $50 \%$ \\
\hline $700-800$ & 5 & 13 & $50 \%$ \\
\hline $800-900$ & 5 & 6 & $50 \%$ \\
\hline $900-1000$ & 5 & 10 & $50 \%$ \\
\hline
\end{tabular}




\subsection{No Active Spacecraft Collisions Scenario}

In this scenario, all parameters are kept the same as in the baseline case except all collisions involving active spacecraft are mitigated. Collisions between inactive spacecraft and other satellite types still occur. This can be considered an upper bound to the benefit that can be derived from implementing space traffic management techniques such as the investigated slot architecture. As the results of Section 5.4 below show, the growth of fragments cannot be tamed without some form of debris removal. Reference [1] states that "by selectively retrieving the most likely future debris sources," objects must be removed at a relatively low rate to curb the predicted growth of fragments.

\subsection{No SSO Collisions Scenario}

Because the model does not track individual satellites, it is impossible to explicitly remove solely the SSO-on-SSO collisions. This effect can instead be simulated via manipulation of the model parameters. By removing the active SSO population during calculation of the spacecraft-on-spacecraft nonuniformity factor, the heightened collision rate near the poles will be lowered by approximately the same amount that implementing the slot architecture with 100\% compliance would achieve. The SSO population is still accounted for in all other calculations including the initial conditions.

The spatial density of rocket bodies and fragments for the entire 500 to 1000 $\mathrm{km}$ altitude region is shown in Figure 5.1 while the density of spacecraft with and without active SSOs is shown in Figure 5.2. The highest density for each of the satellite types occurs at the latitudes between $65^{\circ}$ and $85^{\circ}$. The most significant 
difference in spacecraft density occurs at these same upper latitudes.

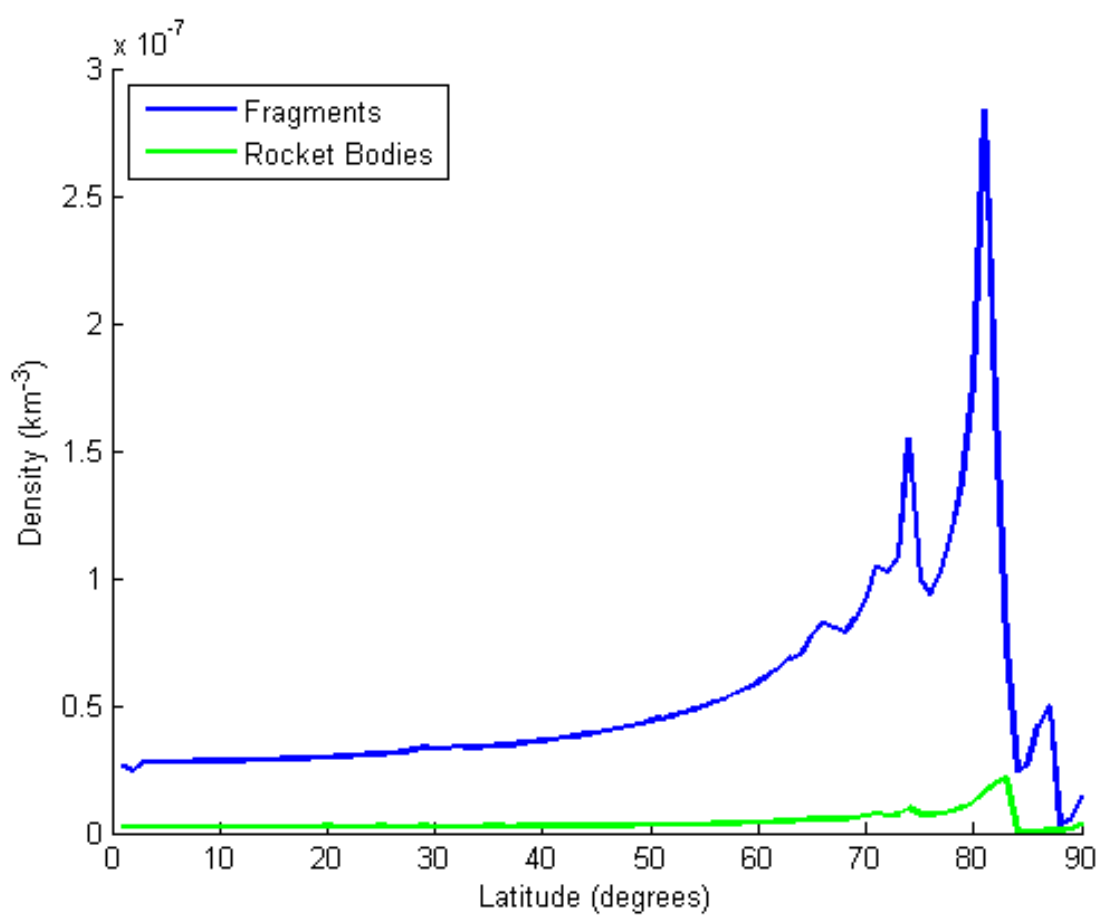

Figure 5.1: Density per latitude bin of the current space environment

\subsection{Results and Discussion}

The results displayed in this section are the sum of the results for each of the six SOI. The slot architecture with full compliance scenario (indicated by "Full SA") reduces the growth of all objects compared to the baseline (businessas-usual) results. The total reduction in spacecraft destroyed is greater than the decrease in intact-on-intact collisions (recall that in this work intacts include rocket bodies and all spacecraft); this is because each intact-on-intact collision produces many new pieces of debris which in turn can destroy spacecraft.

Figure 5.3 shows the number of active spacecraft destroyed for each of the three scenarios outlined. Both post-mission deorbit compliance and number of 


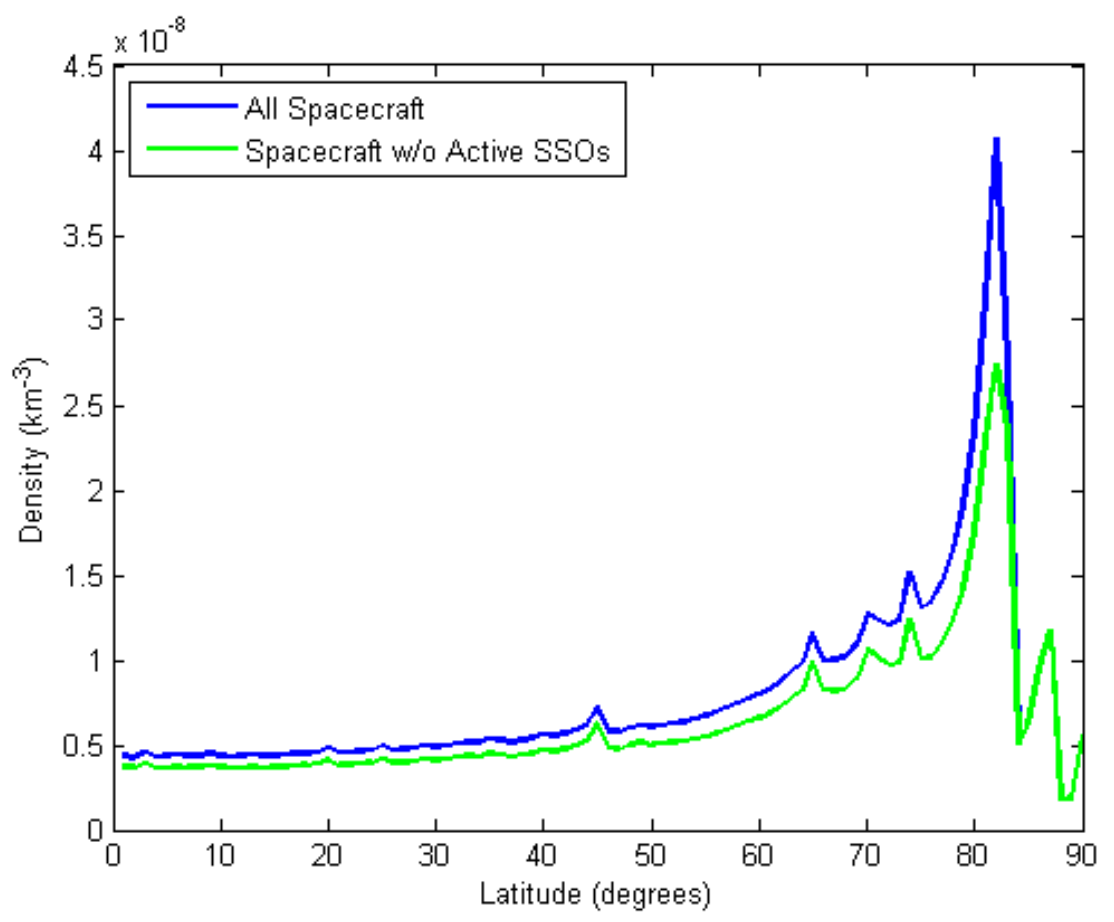

Figure 5.2: Density per latitude bin of the current space environment without active SSO satellites

launches are held constant throughout integration of the model. The collision avoidance success rate is set to $0 \%$ for the baseline and $40 \%$ for the slot architecture scenario. This means that SSO-on-SSO collisions are fully mitigated, and all other collisions involving an active spacecraft have a $40 \%$ chance of being avoided. The figure reveals that relative to the baseline, the perfectly implemented slot architecture scenario reduces the number of active spacecraft destroyed by $58.6 \%$. The reduction in intact-on-intact collisions relative to this baseline is $4.2 \%$ with the slot architecture and $7.2 \%$ with all active satellite collisions avoided. Figure 5.4 shows that similar reductions occur in the growth of fragments; the slot architecture reduces total fragments by $5.1 \%$ and the case with no active spacecraft collisions reduces fragments by $8.8 \%$ relative to the baseline scenario with no collision avoidance. 


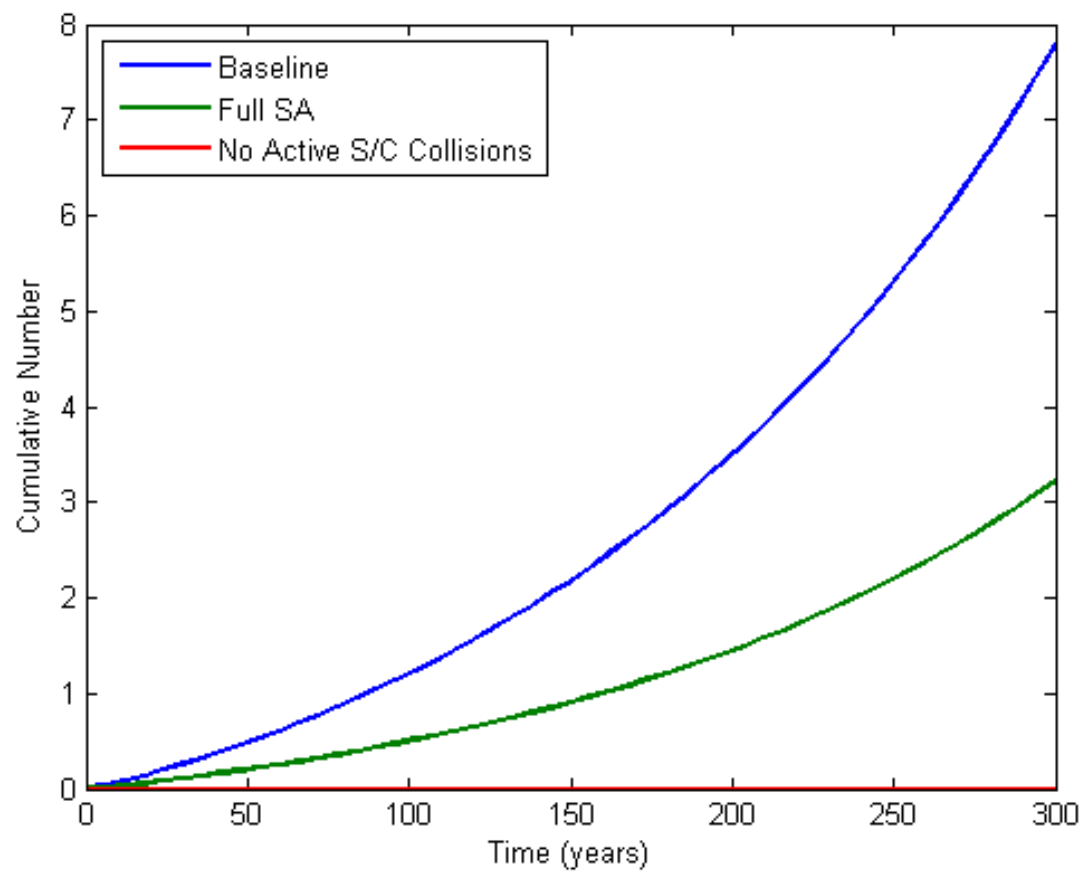

\section{Figure 5.3: Number of intact-on-intact collisions and total number of active spacecraft destroyed}

If the baseline collision avoidance success rate is also set to $40 \%$, the reduction in spacecraft destroyed caused by the slot architecture is $29.9 \%$. Figure 5.5 shows this result. The reduction in intact-on-intact collisions is only $1.3 \%$ for the slot architecture and $4.5 \%$ for the case with no active spacecraft collisions which indicates the vast majority of intact-on-intact collisions involve some combination of rocket bodies and inactive spacecraft. Using the methods described in Section 5.1.1, it is estimated that there are approximately 207 inactive SSO satellites; this is greater than the total number of active satellites in the regime. The efficacy of the architecture is severely limited by the inability of these satellites to deorbit or comply with the architecture.

The evolution of the fragment environment is shown in Figure 5.6. Neither the fully compliant slot architecture nor the mitigation of all collisions involving active 


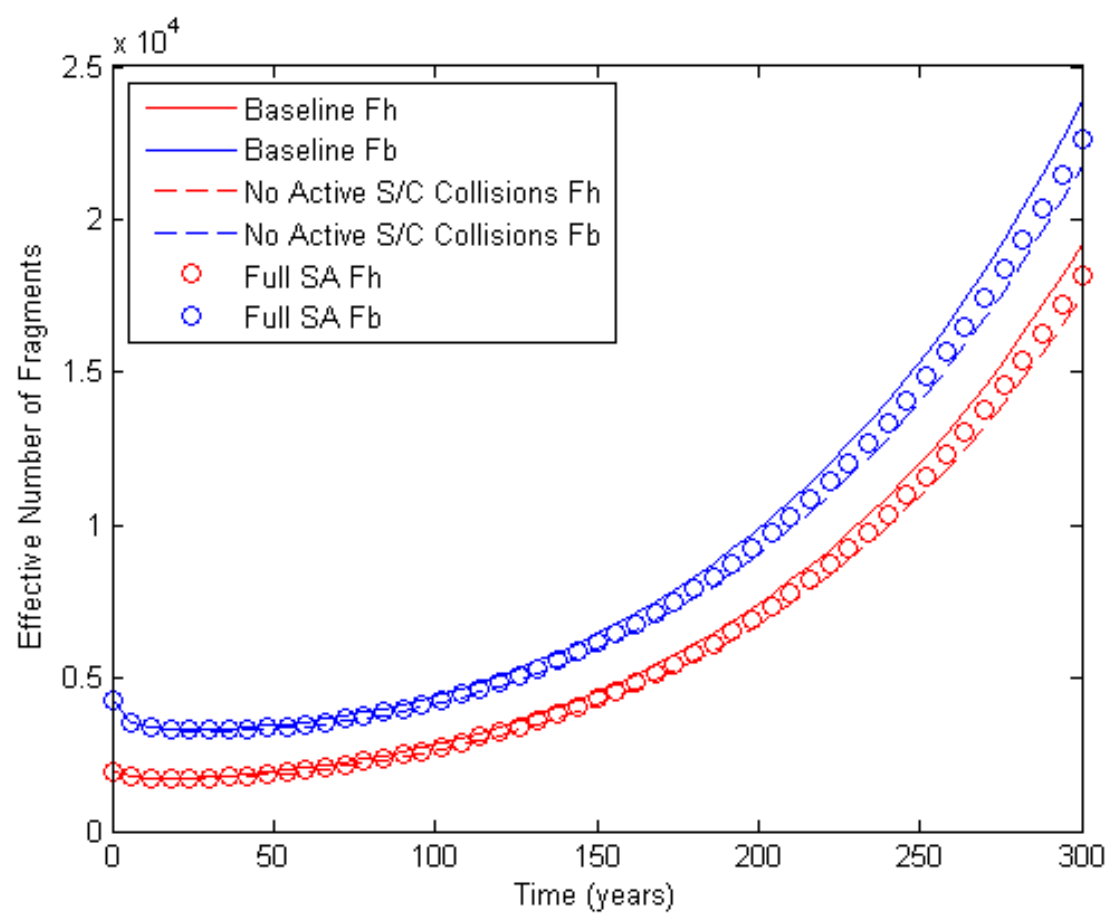

Figure 5.4: Evolution of hazardous and benign fragments

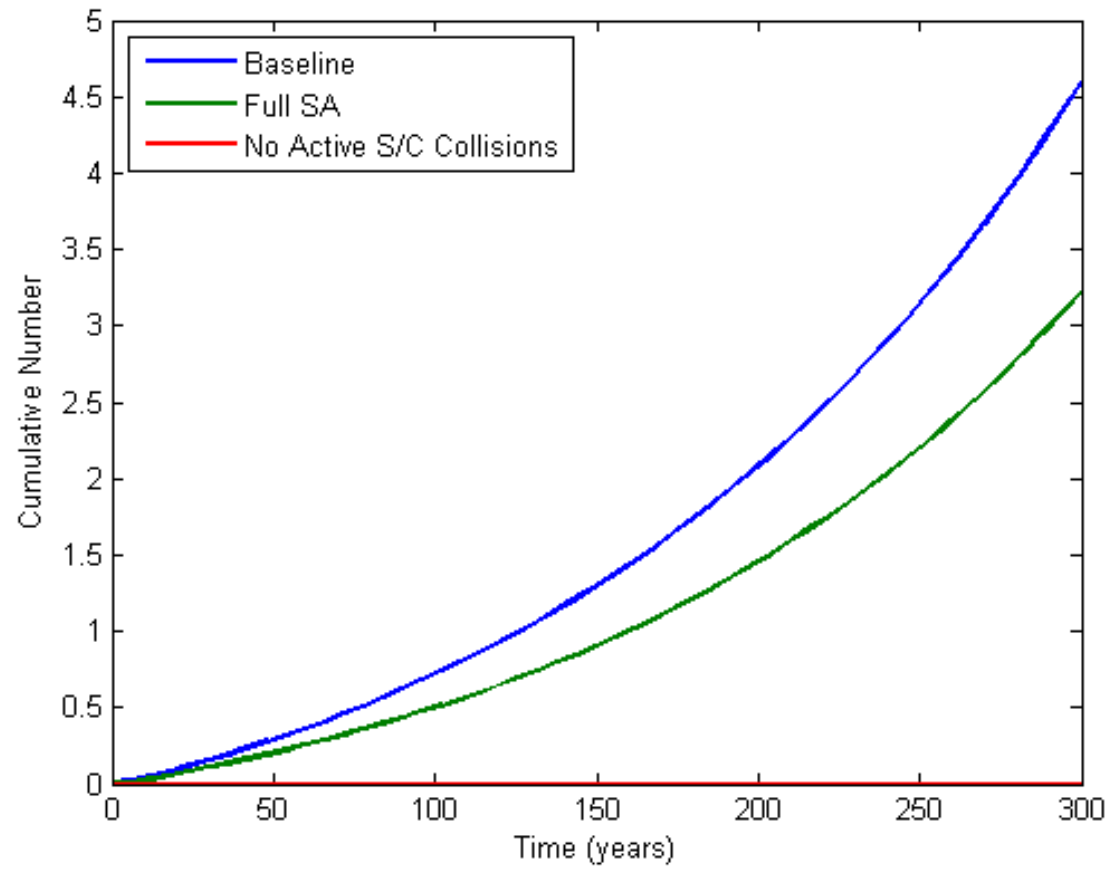

Figure 5.5: Evolution of hazardous and benign fragments 
spacecraft significantly deters the growth of fragments. Relative to the businessas-usual case, the architecture produces a $1.6 \%$ reduction in total fragments while the mitigation of all active spacecraft collisions reduces the number of fragments by $5.4 \%$. This suggests that the majority of collisions resulting in new fragments involve inactive spacecraft, lingering rocket bodies, and other fragments.

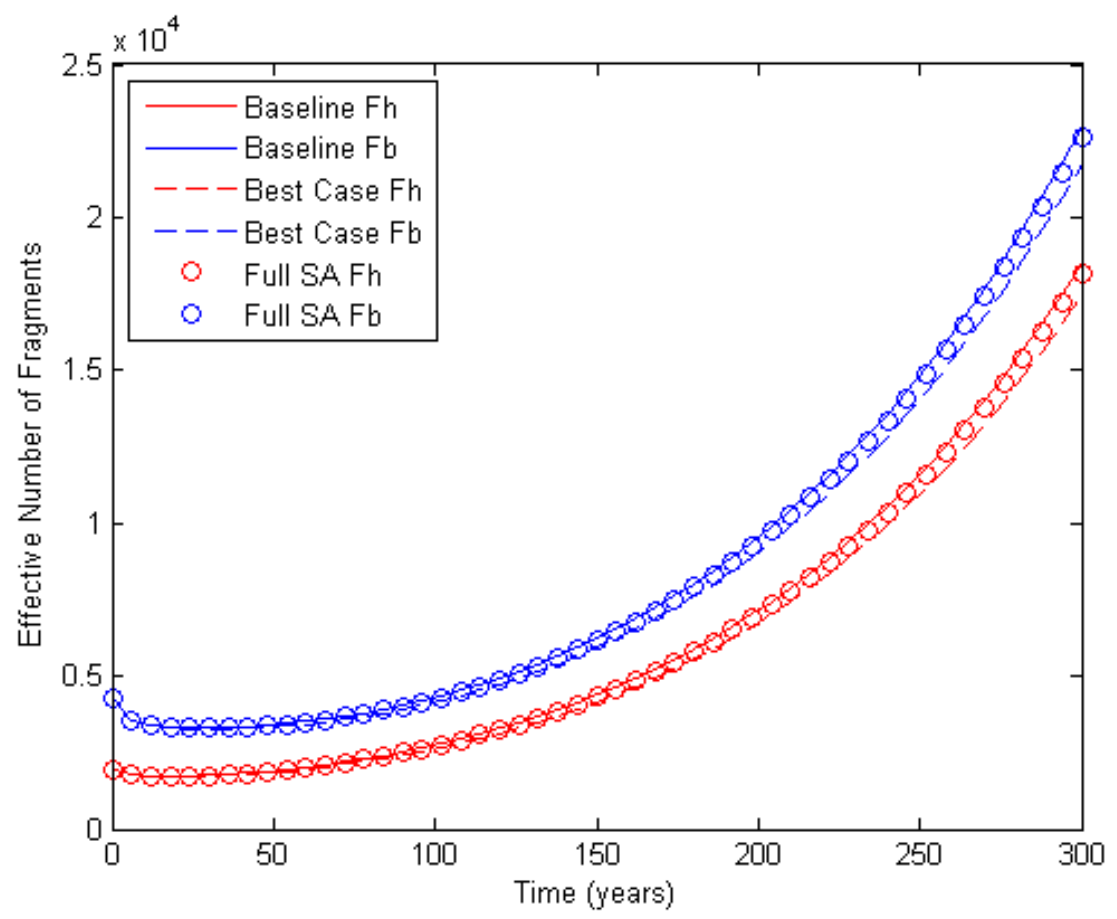

Figure 5.6: Evolution of hazardous and benign fragments

In the limit as the success rate of avoidance maneuvers by active spacecraft approaches 100\%, the efficacy of the architecture is limited to reducing close approaches amongst SSOs. This would require that all active spacecraft have maneuver capability, that all conjunctions are found in time to perform avoidance maneuvers, and that all maneuvers successfully avoid collisions. In this case, the spacecraft destroyed are entirely inactive and the intact-on-intact collisions occur solely between a combination of inactive satellites and rocket bodies. If this were the case, the architecture which intends to keep SSO satellites well-separated 
becomes meaningless.

At the opposite end of the spectrum, the success rate of avoidance maneuvers is set to $0 \%$ for all non-SSO-on-SSO collisions in the slot architecture scenario. The results are shown in Figure 5.7; the slot architecture reduces the number of active spacecraft destroyed by $30.2 \%$ relative to the baseline, and reduces the number of intact-on-intact collisions by $2.1 \%$. Mitigating all collisions involving active spacecraft reduces the number of intact-on-intact collisions by $7.2 \%$.

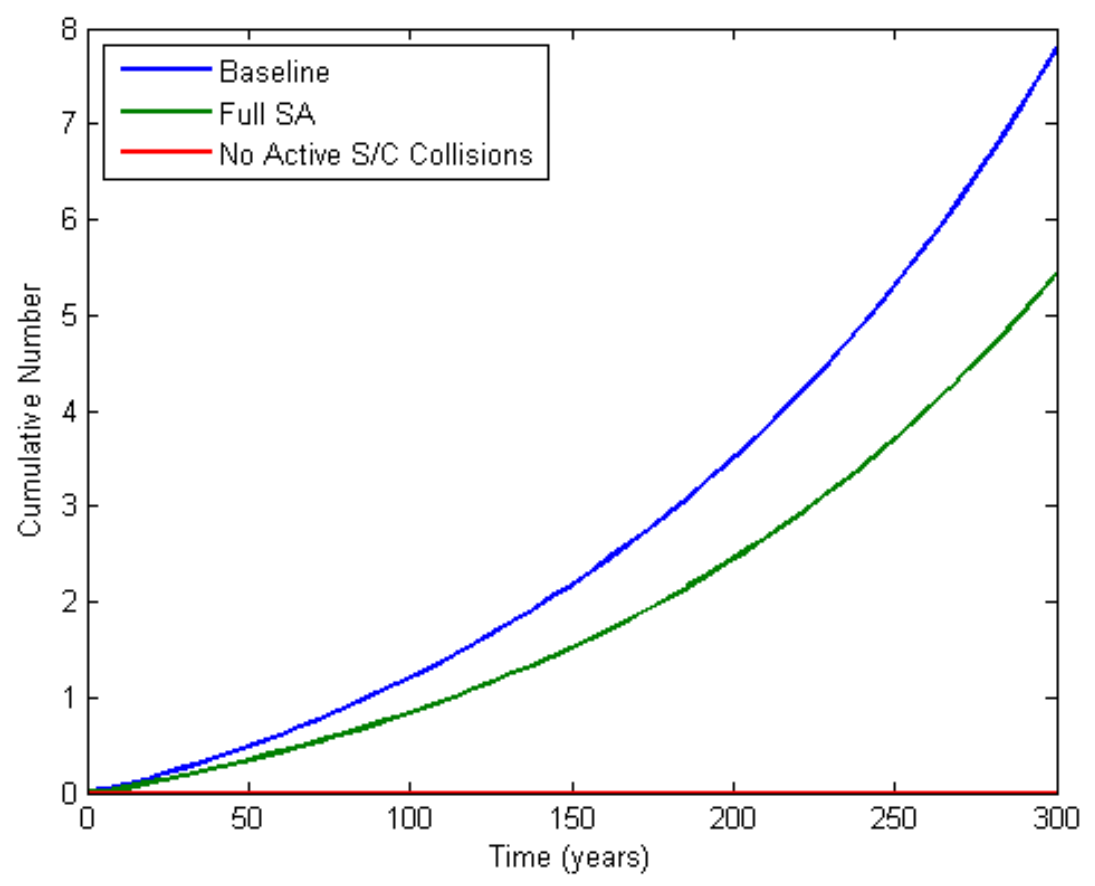

Figure 5.7: Cumulative number of intact-on-intact collisions and spacecraft destroyed with no collision avoidance

The results shown thus far excluded the effects detailed in Section 4.1.3. With an escalating launch rate, the total number of spacecraft increases rapidly and as a consequence, the total number of spacecraft destroyed rises as well. If the post-mission deorbiting compliance rate is held constant (see the values listed in Table 5.1), the impact of the slot architecture diminishes due to the proliferation 
of inactive satellites in SSO. Introducing Equation 4.7 into the model with varying maximum compliance rate reveals that so long as the SSOs deorbit post-mission, the architecture can benefit the environment. Table 5.2 shows the reduction in spacecraft destruction relative to the baseline under various maximum postmission deorbit compliance rates and collision avoidance success rates. Explicitly, the baseline scenario has no collision avoidance while the slot architecture has full avoidance between SSOs and the percentage listed in Table 5.2 for all other collisions involving an active spacecraft. The model is set to asymptotically approach the deorbit compliance values of the first column in the next century and remain constant thereafter. The escalation in launches is set to $1 \%$ of the original launch rate (which varies with SOI) per year.

Table 5.2: Percent reduction in number of active spacecraft destroyed relative to baseline case due to full compliance with the slot architecture for various model configurations

\begin{tabular}{|c|c|c|c|}
\hline $\begin{array}{c}\text { Post-Mission } \\
\text { Deorbit Compliance }\end{array}$ & $\begin{array}{c}\text { 0\% Success of } \\
\text { Avoidance Maneuvers }\end{array}$ & $\begin{array}{c}40 \% \text { Success of } \\
\text { Avoidance Maneuvers }\end{array}$ & $\begin{array}{c}100 \% \text { Success of } \\
\text { Avoidance Maneuvers }\end{array}$ \\
\hline $50 \%$ & $31.4 \%$ & $59.9 \%$ & $100 \%$ \\
\hline $75 \%$ & $66.3 \%$ & $80.3 \%$ & $100 \%$ \\
\hline $90 \%$ & $80.0 \%$ & $88.3 \%$ & $100 \%$ \\
\hline $100 \%$ & $87.0 \%$ & $92.4 \%$ & $100 \%$ \\
\hline
\end{tabular}




\section{Chapter 6}

\section{Concluding Remarks}

At the highest level, the contributions of this work can be split into a refined relative motion analysis, and the development and use of an evolutionary orbital debris model to quantify the effects of a sun-synchronous orbit slot architecture as a form of space traffic management.

Section 3.2 saw the development of a simplistic conformance criterion based on the minimum separation provided by the slot architecture. This criterion is necessary to coarsely judge the amount of time a satellite can comply with the architecture and thereby estimate the altitudes at which the constellation is feasible. Analysis of satellite motion relative to the simplified motion of the proposed slots reveals that the slot architecture may be impractical at altitudes below 500 $\mathrm{km}$. Above $500 \mathrm{~km}$, the conformance times for a satellite with a high drag configuration are on the order of typical times between maintenance maneuvers of high value satellites. Satellites with higher ballistic coefficients (corresponding to a lower drag configuration) can expect longer periods of conformance before a maneuver becomes necessary. 
An evolutionary orbital debris environment model has been adapted to suit the needs of this work. The model consists of coupled ordinary differential equations that attempt to capture much of the complexity of the problem at hand. The results of Chapter 5 indicate that a perfectly implemented slot architecture will reduce the frequency of SSO conjunctions to zero at the cost of performing regular slot maintenance maneuvers.

The model further enforces the idea that although preventive measures like the slot architecture are capable of reducing collisions and thereby slowing the growth of space debris, active debris removal is still required to prevent future growth that will increasingly limit spacecraft capabilities. Only under high compliance with NASA's post-mission deorbit guideline and strategic removal of high probability debris sources can the collisional cascading commonly referred to as the "Kessler syndrome" be prevented.[10, 12]

There were many benefits to the orbital debris model that was utilized, chief among them being computational efficiency and an ability to easily modify the model for new purposes. Nevertheless, use of a high-fidelity, object-by-object model would further elucidate the results of a slot architecture for separating sun-synchronous orbiting satellites due to the ability to explicitly remove SSO collisions rather than merely emulate this effect as done in this work. This would have the additional benefit of determining if there any unintended consequences of the architecture; one such idea being that, with the architecture implemented at many altitudes, the satellites could effectively form a barrier through which launching of future satellites and deorbiting of existing satellites could prove difficult. 


\section{Chapter 7}

\section{Future Work}

There remain a number of hurdles to overcome before anything resembling the proposed slot architecture could be implemented. Putting aside the political and economic concerns, there are still many technical issues to be resolved. If, hypothetically, the architecture were to be implemented in the near future, how would it mesh the current environment? A significant proportion of the current active satellites are not maneuverable and those that are may be unwilling to relocate to the closest available slot as it may impact the satellites' ability to complete their tasks. Additionally, as noted in Reference [7], the optimal vertical stratification (among other parameters) of the slot architecture is both subjective and time-varying. Furthermore, the complexities of inserting new satellites into the architecture as well as post-mission deorbiting from the architecture must be considered.

Although the model developed is likely to be correct in a coarse sense, a highfidelity model should be used to both validate and refine the calculations made herein. Whether an aggregate model like the one used in this work or an objecton-object model would best suit this purpose remains to be seen. Such a model 
could elucidate not only the benefits but also potential detriments of the architecture. Unfortunately these models tend to be proprietary and/or unavailable to the general public so this point is not easily accomplished.

The MATLAB code implementing the model laid out in this work is available to the public through the aerospace engineering department at California Polytechnic State University, San Luis Obispo. The code may be altered and distributed as necessary. 


\section{Bibliography}

[1] Donald J. Kessler, Nicholas L. Johnson, J-C Liou, and Mark Matney. The kessler syndrome: implications to future space operations. Advances in the Astronautical Sciences, 137(8), 2010.

[2] Karl D. Bilimoria and Rogier A. Krieger. Slot architecture for separating satellites in sun-synchronous orbits. In Proceedings of the AIAA SPACE Conference and Exposition, Long Beach, California, USA, volume 1, pages 1110-1122, 2011.

[3] Union of Concerned Scientists. UCS Satellite Database. http://www.ucsusa.org, December 2012.

[4] United States Strategic Command. Space track: The source for space surveillance data. http://www.space-track.org, March 2013.

[5] David A. Vallado. Fundamentals of astrodynamics and applications. Springer-Microcosm Press, New York, 3rd edition, 2007.

[6] Ronald J. Boain. A-b-cs of sun-synchronous orbit mission design. In AAS/AIAA Space Flight Mechanics Conference. Pasadena, CA: Jet Propulsion Laboratory, National Aeronautics and Space Administration, 2004., 2004 . 
[7] Eric Watson. Sun-synchronous orbit slot architecture analysis and development. Master's thesis, California Polytechnic State University, 2012.

[8] Alexander Haagsma. Space traffic management - vertical stratification optimization. Master's thesis, Delft University of Technology, 2012.

[9] Jason Wertz and Wiley Larson. Space Mission Analysis and Design. Springer-Microcosm Press, New York, 3rd edition, 1999.

[10] J-C Liou and Nicholas L. Johnson. Instability of the present leo satellite populations. Advances in Space Research, 41(7):1046-1053, 2008.

[11] Andrew M. Bradley and Lawrence M. Wein. Space debris: Assessing risk and responsibility. Advances in Space Research, 43(9):1372-1390, 2009.

[12] Donald J. Kessler. Critical density of spacecraft in low earth orbit. NASA JSC-28949, NASA Johnson Space Center, Houston, TX, 2000.

[13] A. Rossi, A. Cordelli, P. Farinella, and L. Anselmo. Collisional evolution of the earth's orbital debris cloud. Journal of Geophysical Research: Planets (1991-2012), 99(E11):23195-23210, 1994.

[14] Federal Aviation Administration. Office of commercial space transportation. http://www.faa.gov, 2010.

[15] Space Launch Report. Launch site statistics. http://www.spacelaunchreport.com, December 2012.

[16] National Aeronautics and Space Administration. Process for limiting orbital debris. NASA Technical Standard STD-8719.14A, NASA Headquarters Office of Safety and Mission Assurance, May 2012. 
[17] Frank Morring. USAF satellite-conjunction advisories called inaccurate. Aviation Week and Space Technology, February 2012. 